\title{
Enantioselective Nazarov Reactions Through Catalytic Asymmetric Proton Transfer
}

\author{
Guangxin Liang and Dirk Trauner* \\ Center for New Directions in Organic Synthesis, Department of Chemistry, University of \\ California-Berkeley, Berkeley, CA 94720-1460

\section{Supporting Information}

General. Unless otherwise noted, infrared spectra (IR) were obtained on $\mathrm{NaCl}$ plates with an ATI Mattson Gemini FTIR spectrometer. Proton NMR spectra ( ${ }^{1} \mathrm{H}$ NMR) and carbon NMR spectra $\left({ }^{13} \mathrm{C}\right.$ NMR) were recorded in $\mathrm{CDCl}_{3}$ on either a Bruker AV-400 or a Bruker DRX-500 spectrometer. High-resolution mass spectra (HRMS) were obtained on a VG ProSpec Mass Spectrometer using electron impact (EI) at $70 \mathrm{eV}$ unless otherwise noted. Preparative HPLC was performed on a Varian preparative HPLC instrument with a dynamax Microsorb Si column (ID: $21.4 \mathrm{~mm}$, particle size $8 \mu \mathrm{m}$, length: $25 \mathrm{~cm}$, pore size: $60 \AA$ ) with a linear gradient of $8 \%$ EtOAc in hexanes to $25 \%$ EtOAc in hexanes over a course of $30 \mathrm{~min}$ with a flow rate of $21.6 \mathrm{~mL} / \mathrm{min}$.

All reaction mixtures were magnetically stirred in oven-dried glassware under a blanket of nitrogen. The reaction was performed at room temperature unless otherwise noted. External bath temperatures were used to record all reaction mixture temperatures. Analytical thin layer chromatography (TLC) was carried out on Merck silica gel $60 \mathrm{~F}_{254}$ TLC plates. TLC visualization was accomplished using $254 \mathrm{~nm}$ UV light or charring solutions of $\mathrm{KMnO}_{4}$. Flash column chromatography was performed on ICN siliTech 32$63 \mathrm{D} 60 \AA$ Ailica gel according to the procedure of Still. ${ }^{1}$

Tetrahydrofuran (THF) and dichloromethane $\left(\mathrm{CH}_{2} \mathrm{Cl}_{2}\right)$ were dried according to the procedure described by Bergman. ${ }^{2}$ Acetonitrile $(\mathrm{MeCN})$ was distilled from $\mathrm{CaH}_{2}$ immediately prior to use. Extracts were dried over anhydrous $\mathrm{Na}_{2} \mathrm{SO}_{4}$ and solvents were removed with a rotary evaporator at aspirator pressure.

Preparation of substrates $14, \mathbf{1 7} \mathbf{a}, \mathbf{1 7} \mathbf{b}, \mathbf{1 7} \mathbf{e}$ and $\mathbf{1 7} \mathbf{j}$ and the corresponding cyclization products $15 \mathbf{a}, \mathbf{1 5} \mathbf{b}, \mathbf{1 8 a}, \mathbf{1 8 b}, \mathbf{1 8 e}$ and $\mathbf{1 8 j}$ were reported in our previous paper. ${ }^{3}$ Preparation of substrates $17 \mathbf{c}, 17 \mathbf{d}, 17 \mathbf{f}, 17 \mathrm{~g}, 17 \mathrm{~h}$ and $17 \mathrm{k}$ followed the same strategy and general procedures described in the previous paper.

A general procedure for the catalytic asymmetric Nazarov cyclization: MeCN (4 $\mathrm{mL})$ was added into a mixture of $\mathrm{Sc}(\mathrm{OTf})_{3}(18.0 \mathrm{mg}, 0.0366 \mathrm{mmol}), 16(25.0 \mathrm{mg}, 0.0636$ $\mathrm{mmol})$ and $3 \AA$ molecular sieves $(210.0 \mathrm{mg})$. The reaction mixture was stirred for $80 \mathrm{~min}$ at which time the substrate $(0.366 \mathrm{mmol})$ in $\mathrm{MeCN}(1 \mathrm{~mL})$ was added. When the reaction was complete, the mixture was filtered through Celite and washed with EtOAc $(15 \mathrm{~mL})$. The mixture was concentrated in vacuo and the crude mixture was purified by flash column chromatography. Enantioselectivities were measured on chiral analytical HPLC with either CHIRALPAK ${ }^{\circledR}$ AD $^{\mathrm{TM}}$ column $(250 \mathrm{X} 4.6 \mathrm{~mm})$ or CHIRALPAK $^{\circledR}$ AS column 
$(250 \times 4.6 \mathrm{~mm})$ with a flow rate of $1.0 \mathrm{~mL} / \mathrm{min}$. Integration was done manually and the estimated error of e.e. is $\pm 1 \%$.

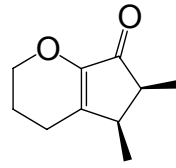

$15 \mathbf{a}$

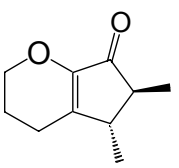

$15 b$

Asymmetric cyclization of $\mathbf{1 4}(61.0 \mathrm{mg}, 0.367 \mathrm{mmol})$ catalyzed by $\mathbf{1 6}$ produced (1 h) $\mathbf{1 5 a}$ and $\mathbf{1 5 b}$ as a diastereomeric mixture. The two diastereomers were separated by preparative HPLC affording enantioenriched 15a $(37.8 \mathrm{mg}, 62.0 \%)$ and 15b (11.0 mg, $18.0 \%)$.

Cis-5,6-Dimethyl-3,4,5,6-tetrahydro-2H-cyclopenta[b]pyran-7-one (15a) The enantiomeric excess was determined by chiral HPLC [AS column, $22 \% i-\mathrm{PrOH}$ in hexanes; $t_{R}($ minor enantiomer $)=7.99 \mathrm{~min}, t_{R}($ major enantiomer $\left.)=9.75 \mathrm{~min}\right]$ to be $40 \%$.

Trans-5,6-Dimethyl-3,4,5,6-tetrahydro-2H-cyclopenta[b]pyran-7-one (15b) The enantiomeric excess was determined by chiral HPLC [AS column, $22 \% i$-PrOH in hexanes; $t_{R}($ minor enantiomer $)=7.79 \mathrm{~min}, t_{R}($ major enantiomer $\left.)=9.40 \mathrm{~min}\right]$ to be $79 \%$.

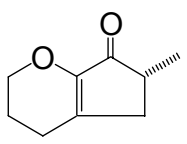

$18 \mathbf{a}$

6-Methyl-3,4,5,6-tetrahydro-2H-cyclopenta[b]pyran-7-one (18a) Asymmetric cyclization of $17 \mathrm{a}(56.0 \mathrm{mg}, 0.366 \mathrm{mmol})$ catalyzed by 16 produced $(3 \mathrm{~h})$ enantioenriched 18a. Purification by flash column chromatography (EtOAc: hexanes $=$ $1: 4)$ afforded 18a $(36.0 \mathrm{mg}, 65 \%)$ as a colorless oil. The enantiomeric excess was determined by chiral HPLC [AS column, $22 \% i$-PrOH in hexanes; $t_{\mathrm{R}}$ (minor enantiomer) $=8.60 \mathrm{~min}, \mathrm{t}_{\mathrm{R}}($ major enantiomer $\left.)=11.35 \mathrm{~min}\right]$ to be $85 \% .[\alpha]_{\mathrm{D}}=-28.6\left(c=1.9, \mathrm{CHCl}_{3}\right)$.

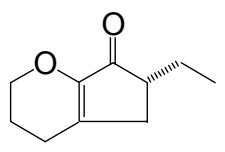

$18 b$

6-Ethyl-3,4,5,6-tetrahydro-2H-cyclopenta[b]pyran-7-one

(18b) Asymmetric cyclization of $\mathbf{1 7 b}(61.0 \mathrm{mg}, 0.366 \mathrm{mmol})$ catalyzed by $\mathbf{1 6}$ produced $(1.5 \mathrm{~h})$ enantioenriched 18b. Purification by flash column chromatography (EtOAc: hexanes $=$ 1:4) afforded 18b (46.0 $\mathrm{mg}, 75 \%)$ as a colorless oil. The enantiomeric excess was determined by chiral HPLC [AS column, $22 \% i-\mathrm{PrOH}$ in hexanes; $\mathrm{t}_{\mathrm{R}}$ (minor enantiomer) $=8.59 \mathrm{~min}, \mathrm{t}_{\mathrm{R}}($ major enantiomer $\left.)=12.04 \mathrm{~min}\right]$ to be $92 \% .[\alpha]_{\mathrm{D}}=-68.8\left(c=1.9, \mathrm{CHCl}_{3}\right)$. 


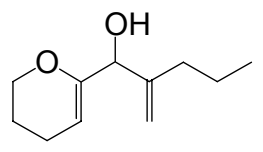

\section{1-(5,6-Dihydro-4H-pyran-2-yl)-2-propyl-prop-2-en-1-ol}

Yield: 91\%; Clear oil; $\mathrm{R}_{f} 0.23$ (EtOAc: hexanes = 1:6); IR 3434(br), 2957, 2930, 2871, 2849, 1675, $1649 \mathrm{~cm}^{-1} ;{ }^{1} \mathrm{H}$ NMR $\left(500 \mathrm{MHz}, \mathrm{CDCl}_{3}\right) \delta_{\mathrm{H}} 5.08(\mathrm{~s}, 1 \mathrm{H}), 4.86(\mathrm{~s}, 1 \mathrm{H}), 4.74$ $(\mathrm{t}, 1 \mathrm{H}, J=3.7 \mathrm{~Hz}), 4.27(\mathrm{~d}, 1 \mathrm{H}, J=6.0 \mathrm{~Hz}), 3.95(\mathrm{~m}, 1 \mathrm{H}), 3.87(\mathrm{~m}, 1 \mathrm{H}), 2.51(\mathrm{~d}, 1 \mathrm{H}, J$ $=6.2 \mathrm{~Hz}), 2.01-1.86(\mathrm{~m}, 4 \mathrm{H}), 1.76-1.68(\mathrm{~m}, 2 \mathrm{H}), 1.45-1.36(\mathrm{~m}, 2 \mathrm{H}), 0.83(\mathrm{t}, 3 \mathrm{H}, J=$ $7.3 \mathrm{~Hz}) ;{ }^{13} \mathrm{C}$ NMR $\left(125 \mathrm{MHz}, \mathrm{CDCl}_{3}\right) \delta_{\mathrm{C}} 153.17,148.58,109.95,97.88,75.21,66.31$, 34.25, 22.23, 20.94, 19.90, 13.83; HRMS calcd for $\mathrm{C}_{11} \mathrm{H}_{18} \mathrm{O}_{2}(\mathrm{M})^{+}$182.1307, found: 182.1304 .

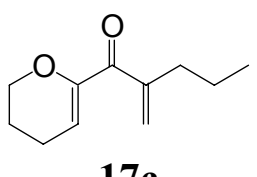

$17 \mathrm{c}$

1-(5,6-Dihydro-4H-pyran-2-yl)-2-propyl-propenone (17c)

Dess-Martin oxidation. Yield: 80\%; Clear oil; $\mathrm{R}_{f} 0.29$ (EtOAc: hexanes $=1: 9$ ); IR 2958, 2932, 2871, 1660, $1623 \mathrm{~cm}^{-1} ;{ }^{1} \mathrm{H}$ NMR (400 MHz, $\left.\mathrm{CDCl}_{3}\right) \delta_{\mathrm{H}} 5.80(\mathrm{t}, 1 \mathrm{H}, J=4.2 \mathrm{~Hz})$, $5.52(\mathrm{~s}, 1 \mathrm{H}), 5.48(\mathrm{~d}, 1 \mathrm{H}, J=1.1 \mathrm{~Hz}), 4.05(\mathrm{t}, 2 \mathrm{H}, J=5.1 \mathrm{~Hz}), 2.25(\mathrm{t}, 2 \mathrm{H}, J=7.5 \mathrm{~Hz})$, $2.16(\mathrm{~m}, 2 \mathrm{H}), 1.81(\mathrm{~m}, 2 \mathrm{H}), 1.36(\mathrm{~m}, 2 \mathrm{H}), 0.84(\mathrm{t}, 3 \mathrm{H}, J=7.3 \mathrm{~Hz}) ;{ }^{13} \mathrm{C}$ NMR $(100$ $\left.\mathrm{MHz}, \mathrm{CDCl}_{3}\right) \delta_{\mathrm{C}} 192.95,151.26,147.35,122.08,114.63,66.32,34.70,21.41,21.12$, 20.90, 13.68; HRMS calcd for $\mathrm{C}_{11} \mathrm{H}_{16} \mathrm{O}_{2}(\mathrm{M})^{+}$180.1150, found: 180.1146 .

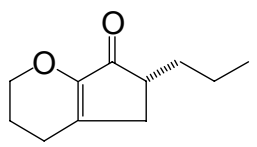

$18 \mathrm{c}$

\section{6-Propyl-3,4,5,6-tetrahydro-2H-cyclopenta[b]pyran-7-one (18c)}

Asymmetric cyclization of 17c $(66.0 \mathrm{mg}, 0.366 \mathrm{mmol})$ catalyzed by 16 produced $(1.5 \mathrm{~h})$ enantioenriched 18c. Purification by flash column chromatography (EtOAc: hexanes = $1: 5)$ afforded 18c $(46.0 \mathrm{mg}, 70 \%)$ as a colorless oil. The enantiomeric excess was determined by chiral HPLC [AS column, $22 \% i$-PrOH in hexanes; $t_{\mathrm{R}}$ (minor enantiomer) $=8.08 \mathrm{~min}, \mathrm{t}_{\mathrm{R}}($ major enantiomer $\left.)=10.71 \mathrm{~min}\right]$ to be $93 \% .[\alpha]_{\mathrm{D}}=-57.7\left(c=2.1, \mathrm{CHCl}_{3}\right)$. $\mathrm{R}_{f} 0.21$ (EtOAc: hexanes = 1:4); IR 2956, 2929, 2872, 1708, $1650 \mathrm{~cm}^{-1} ;{ }^{1} \mathrm{H}$ NMR (400 $\left.\mathrm{MHz} \mathrm{CDCl}_{3}\right) \delta_{\mathrm{H}} 4.08-3.99(\mathrm{~m}, 2 \mathrm{H}), 2.56(\mathrm{dd}, 1 \mathrm{H}, J=17.4,6.3 \mathrm{~Hz}), 2.28(\mathrm{~m}, 3 \mathrm{H}), 2.07$ $(\mathrm{dd}, 1 \mathrm{H}, J=17.4,1.6 \mathrm{~Hz}), 1.88(\mathrm{~m}, 2 \mathrm{H}), 1.79-1.69(\mathrm{~m}, 1 \mathrm{H}), 1.42-1.18(\mathrm{~m}, 3 \mathrm{H}), 0.86(\mathrm{t}$, $3 \mathrm{H}, J=7.2 \mathrm{~Hz}) ;{ }^{13} \mathrm{C}$ NMR $\left(100 \mathrm{MHz}, \mathrm{CDCl}_{3}\right) \delta_{\mathrm{C}} 203.03,150.54,144.19,66.75,43.31$, $33.60,32.79,24.03,21.61,20.27,14.00$; HRMS calcd for $\mathrm{C}_{11} \mathrm{H}_{16} \mathrm{O}_{2}(\mathrm{M})^{+} 180.1150$, found: 180.1148 . 


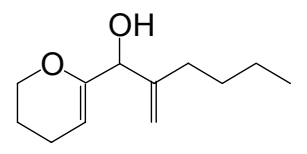

\section{2-Butyl-1-(5,6-dihydro-4H-pyran-2-yl)-prop-2-en-1-ol}

Yield: 82\%; Clear oil; $\mathrm{R}_{f} 0.21$ (EtOAc: hexanes = 1:6); IR 3442(br), 2954, 2929, 2870, $1675,1649 \mathrm{~cm}^{-1} ;{ }^{1} \mathrm{H}$ NMR $\left(500 \mathrm{MHz}, \mathrm{CDCl}_{3}\right) \delta_{\mathrm{H}} 5.14(\mathrm{~s}, 1 \mathrm{H}), 4.94(\mathrm{~s}, 1 \mathrm{H}), 4.81(\mathrm{t}, 1 \mathrm{H}$, $J=3.7 \mathrm{~Hz}), 4.36(\mathrm{~d}, 1 \mathrm{H}, J=6.2 \mathrm{~Hz}), 4.03(\mathrm{~m}, 1 \mathrm{H}), 3.96(\mathrm{~m}, 1 \mathrm{H}), 2.15(\mathrm{~d}, 1 \mathrm{H}, J=6.4$ Hz), 2.06-1.98 (m, $4 \mathrm{H}), 1.79$ (m, $2 \mathrm{H}), 1.43(\mathrm{~m}, 2 \mathrm{H}), 1.30(\mathrm{~m}, 2 \mathrm{H}), 0.88$ (t, $3 \mathrm{H}, J=7.3$ $\mathrm{Hz}) ;{ }^{13} \mathrm{C} \mathrm{NMR}\left(125 \mathrm{MHz}, \mathrm{CDCl}_{3}\right) \delta_{\mathrm{C}} 153.04,148.90,109.88,98.18,75.43,66.46,31.93$, 30.09, 22.48, 22.27, 19.95, 13.94; HRMS calcd for $\mathrm{C}_{12} \mathrm{H}_{20} \mathrm{O}_{2}(\mathrm{M})^{+}$196.1463, found: 196.1465 .

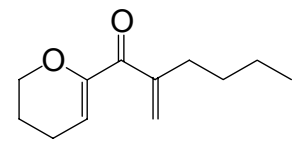

17d

\section{2-Butyl-1-(5,6-dihydro-4H-pyran-2-yl)-propenone (17d)}

Dess-Martin oxidation. Yield: 75\%; Clear oil; $\mathrm{R}_{f} 0.27$ (EtOAc: hexanes = 1:9); IR 2955, 2931, 2872, 1660, $1625 \mathrm{~cm}^{-1} ;{ }^{1} \mathrm{H}$ NMR $\left(500 \mathrm{MHz}, \mathrm{CDCl}_{3}\right) \delta_{\mathrm{H}} 5.83(\mathrm{t}, 1 \mathrm{H}, J=4.1 \mathrm{~Hz})$, $5.54(\mathrm{~s}, 1 \mathrm{H}), 5.51(\mathrm{~s}, 1 \mathrm{H}), 4.08(\mathrm{t}, 2 \mathrm{H}, J=5.1 \mathrm{~Hz}), 2.30(\mathrm{t}, 2 \mathrm{H}, J=7.5 \mathrm{~Hz}), 2.19(\mathrm{dd}, 2$ $\mathrm{H}, J=10.7,6.1 \mathrm{~Hz}), 1.84(\mathrm{~m}, 2 \mathrm{H}), 1.35(\mathrm{~m}, 2 \mathrm{H}), 1.27(\mathrm{~m}, 2 \mathrm{H}), 0.85(\mathrm{t}, 3 \mathrm{H}, J=7.2 \mathrm{~Hz})$; ${ }^{13} \mathrm{C}$ NMR $\left(125 \mathrm{MHz}, \mathrm{CDCl}_{3}\right) \delta_{\mathrm{C}} 192.98,151.24,147.56,121.84,114.63,66.29,32.34$, 30.04, 22.28, 21.37, 20.88, 13.80; HRMS calcd for $\mathrm{C}_{12} \mathrm{H}_{18} \mathrm{O}_{2}(\mathrm{M})^{+}$194.1307, found: 194.1305 .

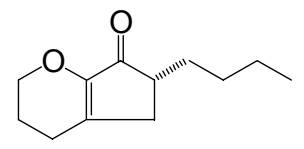

$18 d$

\section{6-Butyl-3,4,5,6-tetrahydro-2H-cyclopenta[b]pyran-7-one (18d)}

Asymmetric cyclization of $\mathbf{1 7 d}(71.0 \mathrm{mg}, 0.366 \mathrm{mmol})$ catalyzed by $\mathbf{1 6}$ produced $(1.5 \mathrm{~h})$ enantioenriched 18d. Purification by flash column chromatography (EtOAc: hexanes $=$ $1: 5)$ afforded 18d $(50.0 \mathrm{mg}, 70 \%)$ as a colorless oil. The enantiomeric excess was determined by chiral HPLC [AS column, $22 \% i$-PrOH in hexanes; $t_{\mathrm{R}}$ (minor enantiomer) $=7.81 \mathrm{~min}, \mathrm{t}_{\mathrm{R}}($ major enantiomer $\left.)=10.55 \mathrm{~min}\right]$ to be $94 \%$. $[\alpha]_{\mathrm{D}}=-56.6\left(c=2.45, \mathrm{CHCl}_{3}\right)$. $\mathrm{R}_{f} 0.21$ (EtOAc: hexanes =1:4); IR 2955, 2927, 2871, 2857, 1710, $1650 \mathrm{~cm}^{-1} ;{ }^{1} \mathrm{H}$ NMR $\left(400 \mathrm{MHz}, \mathrm{CDCl}_{3}\right) \delta_{\mathrm{H}} 4.04(\mathrm{~m}, 2 \mathrm{H}), 2.60-2.54(\mathrm{~m}, 1 \mathrm{H}), 2.29(\mathrm{t}, 3 \mathrm{H}, J=6.2 \mathrm{~Hz}), 2.07$ (dd, $1 \mathrm{H}, J=17.4,1.7 \mathrm{~Hz}), 1.91(\mathrm{~m}, 2 \mathrm{H}), 1.79(\mathrm{~m}, 1 \mathrm{H}), 1.32-1.21(\mathrm{~m}, 5 \mathrm{H}), 0.87-0.80$ $(\mathrm{m}, 3 \mathrm{H}) ;{ }^{13} \mathrm{C}$ NMR $\left(100 \mathrm{MHz}, \mathrm{CDCl}_{3}\right) \delta_{\mathrm{C}} 203.03,150.56,144.21,66.75,43.49,32.79$, $31.15,29.20,24.04,22.65,21.62,13.94$; HRMS calcd for $\mathrm{C}_{12} \mathrm{H}_{18} \mathrm{O}_{2}(\mathrm{M})^{+}$194.1307, found: 194.1304 . 


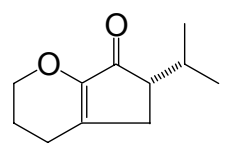

$18 \mathrm{e}$

(S)-6-Isopropyl-3,4,5,6-tetrahydro-2H-cyclopenta[b]pyran-7-one (18e) Asymmetric cyclization of $17 \mathrm{e}(66.0 \mathrm{mg}, 0.366 \mathrm{mmol})$ catalyzed by 16 at $0{ }^{\circ} \mathrm{C}$ produced $(3 \mathrm{~h})$ enantioenriched 18e. Purification by flash column chromatography (EtOAc: hexanes = $1: 5)$ afforded 18e (58.0 $\mathrm{mg}, 88 \%)$ as a colorless oil. The enantiomeric excess was determined by chiral HPLC [AS column, $22 \% i$-PrOH in hexanes; $t_{\mathrm{R}}$ (minor enantiomer) $=7.59 \mathrm{~min}, \mathrm{t}_{\mathrm{R}}($ major enantiomer $\left.)=10.02 \mathrm{~min}\right]$ to be $95 \%$. $[\alpha]_{\mathrm{D}}=-92.2\left(c=2.2, \mathrm{CHCl}_{3}\right)$.

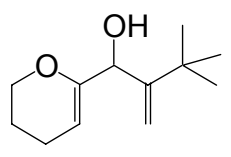

\section{2-tert-Butyl-1-(5,6-dihydro-4H-pyran-2-yl)-prop-2-en-1-ol}

Yield: 92\%; Clear oil; $\mathrm{R}_{f} 0.14$ (EtOAc: hexanes = 1:9); IR 3413(br), 2963, 2932, 2907, 2870, 2849, 1677 $\mathrm{cm}^{-1} ;{ }^{1} \mathrm{H}$ NMR $\left(500 \mathrm{MHz}, \mathrm{CDCl}_{3}\right) \delta_{\mathrm{H}} 5.26(\mathrm{~s}, 1 \mathrm{H}), 5.11(\mathrm{~s}, 1 \mathrm{H}), 4.88(\mathrm{t}$, $1 \mathrm{H}, J=3.7 \mathrm{~Hz}), 4.58$ (d, $1 \mathrm{H}, J=5.6 \mathrm{~Hz}), 4.04-3.93(\mathrm{~m}, 2 \mathrm{H}), 2.09-2.02(\mathrm{~m}, 2 \mathrm{H}), 1.91$ $(\mathrm{d}, 1 \mathrm{H}, J=5.7 \mathrm{~Hz}), 1.80(\mathrm{~m}, 2 \mathrm{H}), 1.11(\mathrm{~s}, 9 \mathrm{H}) ;{ }^{13} \mathrm{C} \mathrm{NMR}\left(125 \mathrm{MHz}, \mathrm{CDCl}_{3}\right) \delta_{\mathrm{C}} 157.87$, 154.87, 109.43, 97.38, 70.88, 66.35, 35.49, 29.44, 22.29, 19.98; HRMS calcd for $\mathrm{C}_{12} \mathrm{H}_{20} \mathrm{O}_{2}(\mathrm{M})^{+}$196.1463, found: 196.1458 .

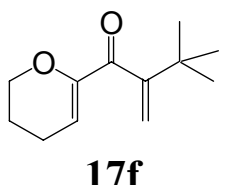

\section{2-tert-Butyl-1-(5,6-dihydro-4H-pyran-2-yl)-propenone (17f)}

Dess-Martin oxidation. Yield: 82\%; Clear oil; $\mathrm{R}_{f} 0.16$ (EtOAc: hexanes $=1: 9$ ); IR 2957, 2910, 2871, 1666, 1625 $\mathrm{cm}^{-1} ;{ }^{1} \mathrm{H}$ NMR $\left(400 \mathrm{MHz}, \mathrm{CDCl}_{3}\right) \delta_{\mathrm{H}} 5.91(\mathrm{t}, 1 \mathrm{H}, J=4.2 \mathrm{~Hz})$, $5.41(\mathrm{~s}, 1 \mathrm{H}), 5.12(\mathrm{~s}, 1 \mathrm{H}), 4.09(\mathrm{t}, 2 \mathrm{H}, J=5.1 \mathrm{~Hz}), 2.25-2.18(\mathrm{~m}, 2 \mathrm{H}), 1.87-1.80(\mathrm{~m}, 2$ $\mathrm{H}), 1.14(\mathrm{~s}, 9 \mathrm{H}) ;{ }^{13} \mathrm{C}$ NMR $\left(100 \mathrm{MHz}, \mathrm{CDCl}_{3}\right) \delta_{\mathrm{C}} 194.77,155.80,152.02,116.89$, 115.69, 66.42, 35.42, 29.63, 21.36, 21.14; HRMS calcd for $\mathrm{C}_{12} \mathrm{H}_{18} \mathrm{O}_{2}(\mathrm{M})^{+}$194.1307, found: 194.1311.

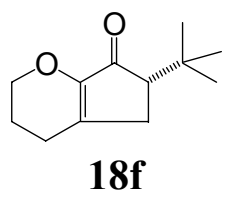

\section{6-tert-Butyl-3,4,5,6-tetrahydro-2H-cyclopenta[b]pyran-7-one (18f)}

Asymmetric cyclization of $17 \mathbf{f}(71.0 \mathrm{mg}, 0.366 \mathrm{mmol})$ catalyzed by 16 produced ( $30 \mathrm{~min})$ enantioenriched 18f. Purification by flash column chromatography (EtOAc: hexanes = 1:6) afforded $18 f(67.0 \mathrm{mg}, 94 \%)$ as a colorless oil. The enantiomeric excess was 
determined by chiral HPLC [AS column, 22\% $i$-PrOH in hexanes; $\mathrm{t}_{\mathrm{R}}$ (minor enantiomer) $=5.52 \mathrm{~min}, \mathrm{t}_{\mathrm{R}}($ major enantiomer $\left.)=7.52 \mathrm{~min}\right]$ to be $97 \% ;[\alpha]_{\mathrm{D}}=-40.5\left(c=3.2, \mathrm{CHCl}_{3}\right)$; $\mathrm{R}_{f} 0.26$ (EtOAc: hexanes =1:4); IR 2954, 2916, 2870, 1705, $1655 \mathrm{~cm}^{-1} ;{ }^{1} \mathrm{H}$ NMR (400 $\left.\mathrm{MHz}, \mathrm{CDCl}_{3}\right) \delta_{\mathrm{H}} 4.05(\mathrm{~m}, 2 \mathrm{H}), 2.41(\mathrm{dd}, 1 \mathrm{H}, J=17.5,6.4 \mathrm{~Hz}), 2.35-2.25(\mathrm{~m}, 2 \mathrm{H}), 2.21$ $(\mathrm{dd}, 1 \mathrm{H}, J=17.5,1.5 \mathrm{~Hz}), 2.11(\mathrm{~d}, 1 \mathrm{H}, J=5.7 \mathrm{~Hz}), 1.90(\mathrm{~m}, 2 \mathrm{H}), 0.96(\mathrm{~s}, 9 \mathrm{H}) ;{ }^{13} \mathrm{C}$ NMR $\left(100 \mathrm{MHz}, \mathrm{CDCl}_{3}\right) \delta_{\mathrm{C}} 201.75,151.32,143.10,66.74,52.76,33.43,29.68,27.31$, 23.87, 21.69; HRMS calcd for $\mathrm{C}_{12} \mathrm{H}_{18} \mathrm{O}_{2}(\mathrm{M})^{+}$194.1307, found: 194.1305.

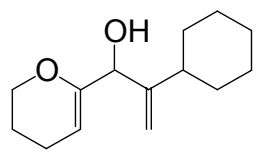

2-Cyclohexyl-1-(5,6-dihydro-4H-pyran-2-yl)-prop-2-en-1-ol

Yield: 75\%; Clear oil; $\mathrm{R}_{f} 0.20$ (EtOAc: hexanes = 1:9); IR 3445(br), 2925, 2850, 1675, $1646 \mathrm{~cm}^{-1} ;{ }^{1} \mathrm{H}$ NMR $\left(400 \mathrm{MHz}, \mathrm{CDCl}_{3}\right) \delta_{\mathrm{H}} 5.14(\mathrm{~s}, 1 \mathrm{H}), 4.93(\mathrm{~s}, 1 \mathrm{H}), 4.80(\mathrm{t}, 1 \mathrm{H}, J=$ $3.7 \mathrm{~Hz}), 4.38(\mathrm{~d}, 1 \mathrm{H}, J=5.7 \mathrm{~Hz}), 3.96(\mathrm{~m}, 2 \mathrm{H}), 2.19(\mathrm{~d}, 1 \mathrm{H}, J=6.2 \mathrm{~Hz}), 2.09-1.94(\mathrm{~m}$, $2 \mathrm{H}), 1.87(\mathrm{t}, 1 \mathrm{H}, J=11.2 \mathrm{~Hz}), 1.81-1.67(\mathrm{~m}, 6 \mathrm{H}), 1.63(\mathrm{~d}, 1 \mathrm{H}, J=11.6 \mathrm{~Hz}), 1.29-1.02$ $(\mathrm{m}, 5 \mathrm{H}) ;{ }^{13} \mathrm{C}$ NMR $\left(100 \mathrm{MHz}, \mathrm{CDCl}_{3}\right) \delta_{\mathrm{C}} 154.51,153.30,108.48,98.13,74.81,66.39$, $40.43,33.76,32.88,26.86,26.77,26.26,22.28,19.97$; HRMS calcd for $\mathrm{C}_{14} \mathrm{H}_{22} \mathrm{O}_{2}(\mathrm{M})^{+}$ 222.1620, found: 222.1623 .

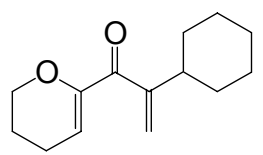

$17 \mathrm{~g}$

2-Cyclohexyl-1-(5,6-dihydro-4H-pyran-2-yl)-propenone (17g)

Dess-Martin oxidation. Yield: 85\%; Clear oil; $\mathrm{R}_{f} 0.25$ (EtOAc: hexanes = 1:9); IR 2926, 2851, 1661, $1625 \mathrm{~cm}^{-1} ;{ }^{1} \mathrm{H}$ NMR $\left(500 \mathrm{MHz}, \mathrm{CDCl}_{3}\right) \delta_{\mathrm{H}} 5.79(\mathrm{t}, 1 \mathrm{H}, J=4.2 \mathrm{~Hz}), 5.33(\mathrm{~s}$, $1 \mathrm{H}), 5.31(\mathrm{~s}, 1 \mathrm{H}), 4.02(\mathrm{t}, 2 \mathrm{H}, J=4.1 \mathrm{~Hz}), 2.41(\mathrm{t}, 1 \mathrm{H}, J=11.6 \mathrm{~Hz}), 2.14(\mathrm{dd}, 2 \mathrm{H}, J=$ 10.7, $6.2 \mathrm{~Hz}), 1.77(\mathrm{~m} 2 \mathrm{H}), 1.69-1.61(\mathrm{~m}, 4 \mathrm{H}), 1.61-1.52(\mathrm{~m}, 1 \mathrm{H}), 1.27-1.14(\mathrm{~m}, 2 \mathrm{H})$, 1.11-1.95 (m, $3 \mathrm{H}) ;{ }^{13} \mathrm{C}$ NMR $\left(125 \mathrm{MHz}, \mathrm{CDCl}_{3}\right) \delta_{\mathrm{C}} 193.84,152.61,151.60,118.15$, 115.63, 66.26, 39.59, 31.63, 26.24, 26.02, 21.29, 20.90; HRMS calcd for $\mathrm{C}_{14} \mathrm{H}_{20} \mathrm{O}_{2}(\mathrm{M})^{+}$ 220.1463, found: 220.1463 .

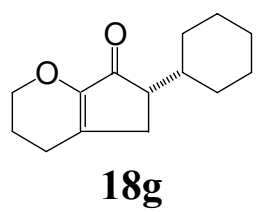

6-Cyclohexyl-3,4,5,6-tetrahydro-2H-cyclopenta[b]pyran-7-one (18g)

Asymmetric cyclization of $\mathbf{1 7 g}(81.0 \mathrm{mg}, 0.366 \mathrm{mmol})$ catalyzed by 16 produced $(3 \mathrm{~h})$ enantioenriched 18g. Purification by flash column chromatography (EtOAc: hexanes $=$ $1: 5)$ afforded $18 \mathrm{~g}(61.0 \mathrm{mg}, 76 \%)$ as a colorless oil. The enantiomeric excess was 
determined by chiral HPLC [AD column, $10 \% i$-PrOH in hexanes; $\mathrm{t}_{\mathrm{R}}$ (minor enantiomer) $=8.56 \mathrm{~min}, \mathrm{t}_{\mathrm{R}}($ major enantiomer $\left.)=12.26 \mathrm{~min}\right]$ to be $76 \% ;[\alpha]_{\mathrm{D}}=-56.2\left(c=2.7, \mathrm{CHCl}_{3}\right)$; $\mathrm{R}_{f} 0.21$ (EtOAc: hexanes $\left.=1: 4\right)$; IR 2922, 2851, 1706, $1650 \mathrm{~cm}^{-1} ;{ }^{1} \mathrm{H}$ NMR $(500 \mathrm{MHz}$, $\left.\mathrm{CDCl}_{3}\right) \delta_{\mathrm{H}} 4.04(\mathrm{~m}, 2 \mathrm{H}), 2.39(\mathrm{dd}, 1 \mathrm{H}, J=17.4,6.3 \mathrm{~Hz}), 2.35-2.24(\mathrm{~m}, 3 \mathrm{H}), 2.21(\mathrm{dd}, 1$ $\mathrm{H}, J=17.4,1.3 \mathrm{~Hz}), 1.94-1.81(\mathrm{~m}, 3 \mathrm{H}), 1.62-1.56(\mathrm{~m}, 4 \mathrm{H}), 1.38(\mathrm{~d}, 1 \mathrm{H}, J=12.7 \mathrm{~Hz})$, $1.20(\mathrm{~m}, 2 \mathrm{H}), 1.11-0.93(\mathrm{~m}, 3 \mathrm{H}) ;{ }^{13} \mathrm{C}$ NMR $\left(125 \mathrm{MHz}, \mathrm{CDCl}_{3}\right) \delta_{\mathrm{C}} 202.63,151.15$, 144.61, 66.68, 48.81, 38.81, 30.65, 28.98, 27.34, 26.42, 26.22, 25.97, 23.96, 21.58; HRMS calcd for $\mathrm{C}_{14} \mathrm{H}_{20} \mathrm{O}_{2}(\mathrm{M})^{+} 220.1463$, found: 220.1464 ; mp: $62.0-64.0{ }^{\circ} \mathrm{C}$ (racemic)

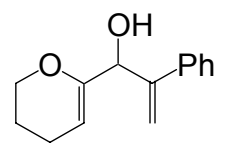

\section{1-(5,6-Dihydro-4H-pyran-2-yl)-2-phenyl-prop-2-en-1-ol}

Yield: 70\%; Clear oil; $\mathrm{R}_{f} 0.24$ (EtOAc: hexanes = 1:6); IR 3441(br), 2929, 2875, 2847, $1675 \mathrm{~cm}^{-1} ;{ }^{1} \mathrm{H}$ NMR $\left(400 \mathrm{MHz}, \mathrm{CDCl}_{3}\right) \delta_{\mathrm{H}} 7.46-7.41(\mathrm{~m}, 2 \mathrm{H}), 7.36-7.27(\mathrm{~m}, 3 \mathrm{H}), 5.53(\mathrm{~s}$, $1 \mathrm{H}), 5.49(\mathrm{~s}, 1 \mathrm{H}), 4.98(\mathrm{~d}, 1 \mathrm{H}, J=5.6 \mathrm{~Hz}), 4.84(\mathrm{t}, 1 \mathrm{H}, J=3.8 \mathrm{~Hz}), 4.02(\mathrm{~m}, 2 \mathrm{H}), 2.34$ $(\mathrm{d}, 1 \mathrm{H}, J=5.9 \mathrm{~Hz}), 2.09-1.93(\mathrm{~m}, 2 \mathrm{H}), 1.86-1.72(\mathrm{~m}, 2 \mathrm{H}) ;{ }^{13} \mathrm{C} \mathrm{NMR}\left(100 \mathrm{MHz}, \mathrm{CDCl}_{3}\right)$ $\delta_{\mathrm{C}} 153.00,148.15,139.73,128.14,127.51,126.89,114.21,98.99,74.12,66.53,22.25$, 19.98; HRMS calcd for $\mathrm{C}_{14} \mathrm{H}_{16} \mathrm{O}_{2}(\mathrm{M})^{+} 216.1150$, found: 216.1149 .

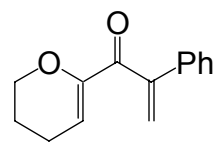

$17 \mathrm{~h}$

\section{1-(5,6-Dihydro-4H-pyran-2-yl)-2-phenyl-propenone (17h)}

Dess-Martin oxidation. Yield: 65\%; Clear oil; $\mathrm{R}_{f} 0.27$ (EtOAc: hexanes $=1: 6$ ); IR 2934, $2875,1666,1623 \mathrm{~cm}^{-1} ;{ }^{1} \mathrm{H}$ NMR $\left(500 \mathrm{MHz}, \mathrm{CDCl}_{3}\right) \delta_{\mathrm{H}}$ 7.39-7.35 (m, $\left.2 \mathrm{H}\right), 7.35-7.27(\mathrm{~m}$, $3 \mathrm{H}), 6.01(\mathrm{t}, 1 \mathrm{H}, J=4.2 \mathrm{~Hz}), 5.87(\mathrm{~s}, 1 \mathrm{H}), 5.55(\mathrm{~s}, 1 \mathrm{H}), 4.10(\mathrm{t}, 2 \mathrm{H}, J=5.1 \mathrm{~Hz}), 2.24-$ $2.14(\mathrm{~m}, 2 \mathrm{H}), 1.85(\mathrm{~m}, 2 \mathrm{H}) ;{ }^{13} \mathrm{C} \mathrm{NMR}\left(125 \mathrm{MHz}, \mathrm{CDCl}_{3}\right) \delta_{\mathrm{C}} 192.58,151.46,147.12$, $136.83,128.52,128.31,126.50,119.08,117.64,66.41,21.28,21.09$; HRMS calcd for $\mathrm{C}_{14} \mathrm{H}_{14} \mathrm{O}_{2}(\mathrm{M})^{+}$214.0994, found: 214.0991 .

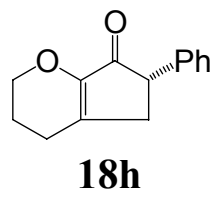

\section{6-Phenyl-3,4,5,6-tetrahydro-2H-cyclopenta[b]pyran-7-one (18h)}

Asymmetric cyclization of $\mathbf{1 7 h}(78.0 \mathrm{mg}, 0.366 \mathrm{mmol})$ catalyzed by $\mathbf{1 6}$ produced $(1 \mathrm{~h})$ enantioenriched 18h. Purification by flash column chromatography (EtOAc: hexanes $=$ $1: 3)$ afforded $18 \mathrm{~h}(51.0 \mathrm{mg}, 65 \%)$ as a white solid. The enantiomeric excess was determined by chiral HPLC [AS column, 35\% $i$-PrOH in hexanes; $\mathrm{t}_{\mathrm{R}}$ (minor enantiomer) $=12.38 \mathrm{~min}, \mathrm{t}_{\mathrm{R}}($ major enantiomer $\left.)=16.04 \mathrm{~min}\right]$ to be $87 \% ;[\alpha]_{\mathrm{D}}=+71.0(c=2.1$, $\mathrm{CHCl}_{3}$ ); $\mathrm{R}_{f} 0.15$ (EtOAc: hexanes = 1:3); IR 2943, 2875, 1705, 1644cm ${ }^{-1} ;{ }^{1} \mathrm{H}$ NMR $(500$ 
$\left.\mathrm{MHz}, \mathrm{CDCl}_{3}\right) \delta_{\mathrm{H}}$ 7.33-7.27 (m, $\left.2 \mathrm{H}\right), 7.25-7.20(\mathrm{~m}, 1 \mathrm{H}), 7.17-7.12(\mathrm{~m}, 2 \mathrm{H}), 4.16(\mathrm{t}, 2 \mathrm{H}$, $J=5.2 \mathrm{~Hz}), 3.56(\mathrm{~d}, 1 \mathrm{H}, J=6.0 \mathrm{~Hz}), 2.99(\mathrm{dd}, 1 \mathrm{H}, J=17.7,6.8 \mathrm{~Hz}), 2.53(\mathrm{dd}, 1 \mathrm{H}, J=$ 17.7, $1.7 \mathrm{~Hz}), 2.42(\mathrm{~m}, 2 \mathrm{H}), 2.06-1.95(\mathrm{~m}, 2 \mathrm{H}),{ }^{13} \mathrm{C} \mathrm{NMR}\left(125 \mathrm{MHz}, \mathrm{CDCl}_{3}\right) \delta_{\mathrm{C}} 200.01$, $150.33,144.84,139.56,128.73,127.65,126.91,66.90,49.33,35.91,24.09,21.54$; HRMS calcd for $\mathrm{C}_{14} \mathrm{H}_{14} \mathrm{O}_{2}(\mathrm{M})^{+} 214.0994$, found: 214.0994 . mp: $117.2-119.0{ }^{\circ} \mathrm{C}$

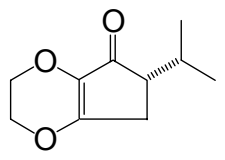

18j

\section{6-Isopropyl-2,3,6,7-tetrahydro-cyclopenta[1,4]dioxin-5-one (10t)}

Asymmetric cyclization of $\mathbf{1 7 j}(67.0 \mathrm{mg}, 0.366 \mathrm{mmol})$ catalyzed by 16 at $0{ }^{\circ} \mathrm{C}$ produced $(3 \mathrm{~h})$ enantioenriched 18j. Purification by flash column chromatography (EtOAc: hexanes $=1: 2)$ afforded 18j $(43.0 \mathrm{mg}, 65 \%)$ as a colorless oil. The enantiomeric excess was determined by chiral HPLC [AS column, 35\% $i$-PrOH in hexanes; $\mathrm{t}_{\mathrm{R}}$ (minor enantiomer) $=8.83 \mathrm{~min}, \mathrm{t}_{\mathrm{R}}($ major enantiomer $\left.)=11.93 \mathrm{~min}\right]$ to be $72 \% ;[\alpha]_{\mathrm{D}}=-60.2\left(c=2.0, \mathrm{CHCl}_{3}\right)$; $\mathrm{R}_{f} 0.22$ (EtOAc: hexanes $=1: 2$ ); IR 2957, 2930, 2873, 1707, $1641 \mathrm{~cm}^{-1} ;{ }^{1} \mathrm{H}$ NMR $\delta_{\mathrm{H}} 4.30$ (m, $2 \mathrm{H}), 4.12$ (t, $2 \mathrm{H}, J=4.0 \mathrm{~Hz}), 2.48(\mathrm{dd}, 1 \mathrm{H}, J=17.0,6.6 \mathrm{~Hz}), 2.40(\mathrm{~m}, 1 \mathrm{H}), 2.28(\mathrm{~m}$, $2 \mathrm{H}), 0.95(\mathrm{~d}, 3 \mathrm{H}, J=7.0 \mathrm{~Hz}), 0.76(\mathrm{~d}, 3 \mathrm{H}, J=6.8 \mathrm{~Hz}) ;{ }^{13} \mathrm{C}$ NMR $\delta_{\mathrm{C}} 196.92,165.08$, 134.31, 66.88, 63.84, 47.66, 28.06, 24.78, 20.40, 16.54; HRMS calcd for $\mathrm{C}_{10} \mathrm{H}_{14} \mathrm{O}_{3}(\mathrm{M})^{+}$ 182.0943, found: 182.0944 .

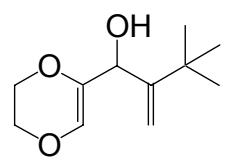

\section{2-tert-Butyl-1-(5,6-dihydro-[1,4]dioxin-2-yl)-prop-2-en-1-ol}

Yield: 70\%; Clear oil; $\mathrm{R}_{f} 0.27$ (EtOAc: hexanes = 1:3); IR 3420(br), 2965, 2936, 2908, 2874, $1680 \mathrm{~cm}^{-1} ;{ }^{1} \mathrm{H}$ NMR $\left(400 \mathrm{MHz}, \mathrm{CDCl}_{3}\right) \delta_{\mathrm{H}} 6.12(\mathrm{~s}, 1 \mathrm{H}), 5.30(\mathrm{~s}, 1 \mathrm{H}), 5.15(\mathrm{~s}, 1 \mathrm{H})$, 4.62 (d, $1 \mathrm{H}, J=4.5 \mathrm{~Hz}), 4.13-4.02(\mathrm{~m}, 2 \mathrm{H}), 4.02-3.95(\mathrm{~m}, 2 \mathrm{H}), 1.97$ (d, $1 \mathrm{H}, J=4.3 \mathrm{~Hz}$ ), $1.09(\mathrm{~s}, 9 \mathrm{H}) ;{ }^{13} \mathrm{C}$ NMR $\left(100 \mathrm{MHz}, \mathrm{CDCl}_{3}\right) \delta_{\mathrm{C}} 156.68,138.25,125.29,110.08,68.90$, 64.55, 63.99, 35.44, 29.39; HRMS calcd for $\mathrm{C}_{11} \mathrm{H}_{18} \mathrm{O}_{3}(\mathrm{M})^{+}$198.1256, found: 198.1253.

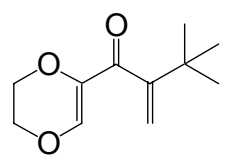

$17 \mathbf{k}$

\section{2-tert-Butyl-1-(5,6-dihydro-[1,4]dioxin-2-yl)-propenone (17k)}

Dess-Martin oxidation. Yield: 30\%; Clear oil; $\mathrm{R}_{f} 0.27$ (EtOAc: hexanes = 1:3); IR 2959, 2906, 2875, 1654, $1611 \mathrm{~cm}^{-1} ;{ }^{1} \mathrm{H}$ NMR $\left(400 \mathrm{MHz}, \mathrm{CDCl}_{3}\right) \delta_{\mathrm{H}} 7.11(\mathrm{~s}, 1 \mathrm{H}), 5.37(\mathrm{~s}, 1 \mathrm{H})$, $5.10(\mathrm{~s}, 1 \mathrm{H}), 4.15(\mathrm{~s}, 4 \mathrm{H}), 1.14(\mathrm{~s}, 9 \mathrm{H}) ;{ }^{13} \mathrm{C} \mathrm{NMR}\left(100 \mathrm{MHz}, \mathrm{CDCl}_{3}\right) \delta_{\mathrm{C}} 192.34,155.48$, 143.07, 137.89, 115.39, 65.11, 63.47, 35.38, 29.64; HRMS calcd for $\mathrm{C}_{11} \mathrm{H}_{16} \mathrm{O}_{3}(\mathrm{M})^{+}$ 196.1100, found: 196.1098 . 


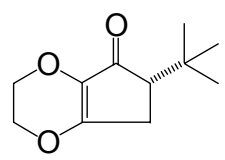

$18 \mathrm{k}$

6-tert-Butyl-2,3,6,7-tetrahydro-cyclopenta[1,4]dioxin-5-one (18k)

Asymmetric cyclization of $\mathbf{1 7 k}(72.0 \mathrm{mg}, 0.366 \mathrm{mmol})$ catalyzed by $\mathbf{1 6}$ produced (30 min) enantioenriched 18k. Purification by flash column chromatography (EtOAc: hexanes = $1: 2)$ afforded $18 \mathrm{k}(57.0 \mathrm{mg}, 80 \%)$ as a colorless oil. The enantiomeric excess was determined by chiral HPLC [AS column, $35 \% i$-PrOH in hexanes; $t_{\mathrm{R}}$ (minor enantiomer) $=5.54 \mathrm{~min}, \mathrm{t}_{\mathrm{R}}$ (major enantiomer $\left.)=7.76 \mathrm{~min}\right]$ to be $91 \% ;[\alpha]_{\mathrm{D}}=-35.6(c=1.6$, $\mathrm{CHCl}_{3}$ ); $\mathrm{R}_{f} 0.12$ (EtOAc: hexanes = 1:3); IR 2955, 2907, 2871, 1706, $1648 \mathrm{~cm}^{-1} ;{ }^{1} \mathrm{H}$ NMR $\left(400 \mathrm{MHz}, \mathrm{CDCl}_{3}\right) \delta_{\mathrm{H}} 4.29(\mathrm{~m}, 2 \mathrm{H}), 4.10(\mathrm{t}, 2 \mathrm{H}, J=4.0 \mathrm{~Hz}), 2.51(\mathrm{dd}, 1 \mathrm{H}, J=17.3,6.7$ $\mathrm{Hz}), 2.31(\mathrm{dd}, 1 \mathrm{H}, J=17.3,2.2 \mathrm{~Hz}), 2.20(\mathrm{dd}, 1 \mathrm{H}, J=6.7,2.2 \mathrm{~Hz}), 0.98(\mathrm{~s}, 9 \mathrm{H}) ;{ }^{13} \mathrm{C}$ NMR $\left(100 \mathrm{MHz}, \mathrm{CDCl}_{3}\right) \delta_{\mathrm{C}} 196.25,163.92,134.28,66.86,63.90,51.45,33.24,27.32$, 26.45; HRMS calcd for $\mathrm{C}_{11} \mathrm{H}_{16} \mathrm{O}_{3}(\mathrm{M})^{+}$196.1100, found: 196.1100 .

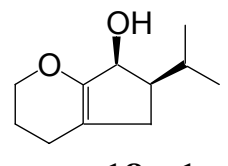

ent-18e-1

$(6 R, 7 S)$-6-Isopropyl-2,3,4,5,6,7-hexahydro-cyclopenta[b]pyran-7-ol (ent-18e-1)

To $0.66 \mathrm{~mL}$ lithium tri-sec-butylborohydride solution (1M in THF) was added $5 \mathrm{~mL}$ THF and the solution was cooled to $0{ }^{\circ} \mathrm{C}$. Then $0.10 \mathrm{~g}(0.55 \mathrm{mmol})$ of ent-18e $(94 \%$ e.e. $)$ in 5 $\mathrm{mL}$ THF, was added. The reaction mixture was stirred vigorously for $0.5 \mathrm{~h}$ at $0{ }^{\circ} \mathrm{C}$ and then allowed to warm to r.t. To the reaction mixture was added $0.5 \mathrm{~mL}$ of water, $1 \mathrm{~mL}$ of ethanol, $0.5 \mathrm{~mL}$ of $30 \%$ hydrogen peroxide and $0.5 \mathrm{~mL}$ of $6 \mathrm{M}$ sodium hydroxide solution. The reaction mixture was diluted with $10 \mathrm{~mL}$ of brine and $20 \mathrm{~mL}$ of EtOAc. The two layers were separated and the aqueous layer was extracted with EtOAc (2 X $10 \mathrm{~mL})$. The combined organic layers were washed with brine $(30 \mathrm{~mL})$, dried, filtered and concentrated in vacuo. The product was purified by flash column chromatography (EtOAc: hexanes $=1: 6)$ to afford $0.095 \mathrm{~g}(95 \%)$ of ent-18e-1 as a clear oil; $\mathrm{R}_{f} 0.15$ (EtOAc: hexanes = 1:6); IR 3349(br), 2952, 2918, 2880, 2867, 2833, $1692 \mathrm{~cm}^{-1}$; ${ }^{1} \mathrm{H}$ NMR $\left(500 \mathrm{MHz}, \mathrm{CDCl}_{3}\right) \delta_{\mathrm{H}} 4.34(\mathrm{~d}, 1 \mathrm{H}, J=5.8 \mathrm{~Hz}), 3.99(\mathrm{~m}, 2 \mathrm{H}), 2.10(\mathrm{~m}, 2 \mathrm{H}), 1.97(\mathrm{~m}, 2$ $\mathrm{H}), 1.82(\mathrm{~m}, 2 \mathrm{H}), 1.76(\mathrm{~m}, 1 \mathrm{H}), 1.65(\mathrm{~m}, 2 \mathrm{H}), 0.99(\mathrm{~d}, 3 \mathrm{H}, J=6.4 \mathrm{~Hz}), 0.88(\mathrm{~d}, 3 \mathrm{H}, J$ $=6.6 \mathrm{~Hz}) ;{ }^{13} \mathrm{C} \mathrm{NMR}\left(125 \mathrm{MHz}, \mathrm{CDCl}_{3}\right) \delta_{\mathrm{C}} 152.92,112.25,74.22,67.05,48.33,35.25$, 27.47, 22.55, 21.97, 21.70, 20.83; HRMS calcd for $\mathrm{C}_{11} \mathrm{H}_{18} \mathrm{O}_{2}(\mathrm{M})^{+}$182.1307, found: 182.1308; $[\alpha]_{\mathrm{D}}=-75.2\left(c=3.7, \mathrm{CHCl}_{3}\right)$; mp: $60.8-62.0{ }^{\circ} \mathrm{C}$ (Racemic). 


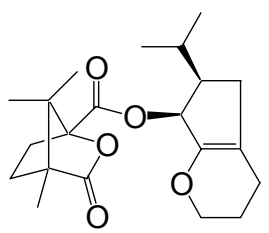

19

\section{4,7,7-Trimethyl-3-oxo-2-oxa-bicyclo[2.2.1]heptane-1-carboxylic acid 6-isopropyl- $\mathbf{2 , 3 , 4 , 5 , 6 , 7 - h e x a h y d r o - c y c l o p e n t a [ b ] p y r a n - 7 - y l ~ e s t e r ~ ( 1 9 ) ~}$}

To a mixture of ent-18e-1 (0.052 g, $0.285 \mathrm{mmol})$, (-)-camphanoyl chloride (0.074 g, $0.342 \mathrm{mmol})$ and DMAP $(0.052 \mathrm{~g}, 0.428 \mathrm{mmol})$ was added $\mathrm{CH}_{2} \mathrm{Cl}_{2}(8 \mathrm{~mL})$. The reaction mixture was stirred for $10 \mathrm{~min}$ before water $(10 \mathrm{~mL})$ was added. The reaction mixture was diluted with $\mathrm{CH}_{2} \mathrm{Cl}_{2}(20 \mathrm{~mL})$ and the two layers were separated. The organic layer was washed with brine $(10 \mathrm{~mL})$ dried, filtered and concentrated in vacuo. The product was purified by flash column chromatography (EtOAc: hexanes $=1: 6$ ) to afford $0.078 \mathrm{~g}$ (75\%) of 19 as a white solid; $\mathrm{R}_{f} 0.21$ (EtOAc: hexanes = 1:6); IR 2965, 2927, 2870, 2847, $1787,1743,1690 \mathrm{~cm}^{-1} ;{ }^{1} \mathrm{H}$ NMR $\left(400 \mathrm{MHz}, \mathrm{CDCl}_{3}\right) \delta_{\mathrm{H}} 5.81(\mathrm{~d}, 1 \mathrm{H}, J=6.1 \mathrm{~Hz}), 4.05-$ 3.95 (m, $1 \mathrm{H}), 3.94-3.85(\mathrm{~m}, 1 \mathrm{H}), 2.39(\mathrm{~m}, 1 \mathrm{H}), 2.27-2.11(\mathrm{~m}, 2 \mathrm{H}), 2.09-1.97(\mathrm{~m}, 3 \mathrm{H})$, 1.97-1.61 (m, $6 \mathrm{H}), 1.09$ (s, $3 \mathrm{H}), 1.03$ (s, $3 \mathrm{H}), 0.95$ (s, $3 \mathrm{H}), 0.90$ (d, $3 \mathrm{H}, J=6.5 \mathrm{~Hz})$, $0.86(\mathrm{~d}, 3 \mathrm{H}, J=6.3 \mathrm{~Hz}) ;{ }^{13} \mathrm{C} \mathrm{NMR}\left(100 \mathrm{MHz}, \mathrm{CDCl}_{3}\right) \delta_{\mathrm{C}} 178.35,167.08,149.55$, $115.58,91.35,77.47,67.04,54.79,54.18,46.82$, 35.54, 30.68, 29.18, 27.66, 22.45, 21.91, 21.84, 20.76, 16.89, 16.48, 9.71; HRMS calcd for $\mathrm{C}_{21} \mathrm{H}_{30} \mathrm{O}_{5}(\mathrm{M})^{+}$362.2093, found: 362.2087; mp: $107-108^{\circ} \mathrm{C} ;[\alpha]_{\mathrm{D}}=-143.0\left(c=0.6, \mathrm{CHCl}_{3}\right)$.

1 Still, W. C.; Kahn, M.; Mitra, A. J. Org. Chem. 1978, 43, 2923-2925.

2 Alaimo, P. J.; Peters, D. W.;Arnold, J.; Bergman, R. G. J. Chem. Ed. 2001, 78, 64. 3 Liang, G.; Gradl, S. N.; Trauner, D. Org. Lett. 2003, 5, 4931-4934. 


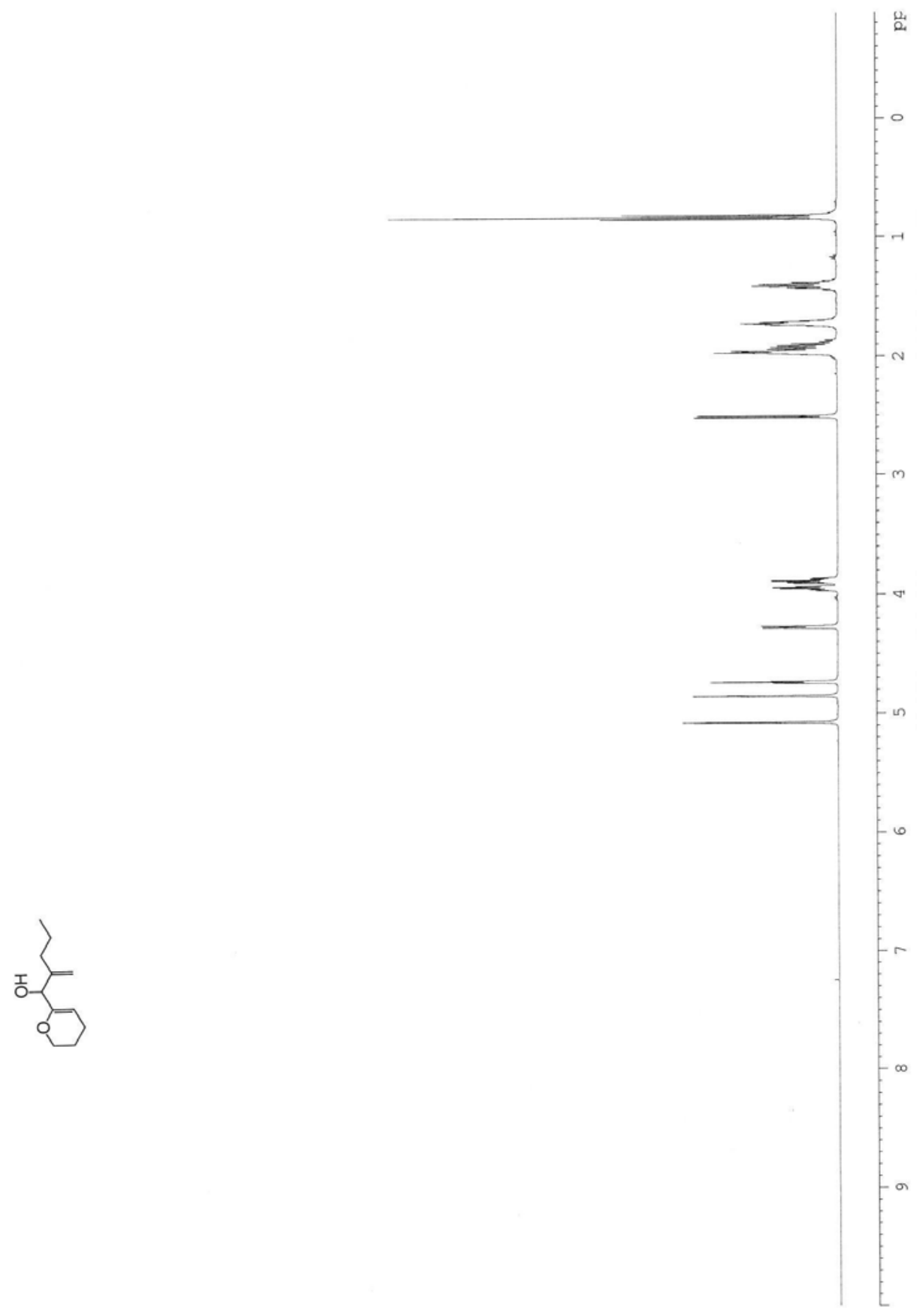




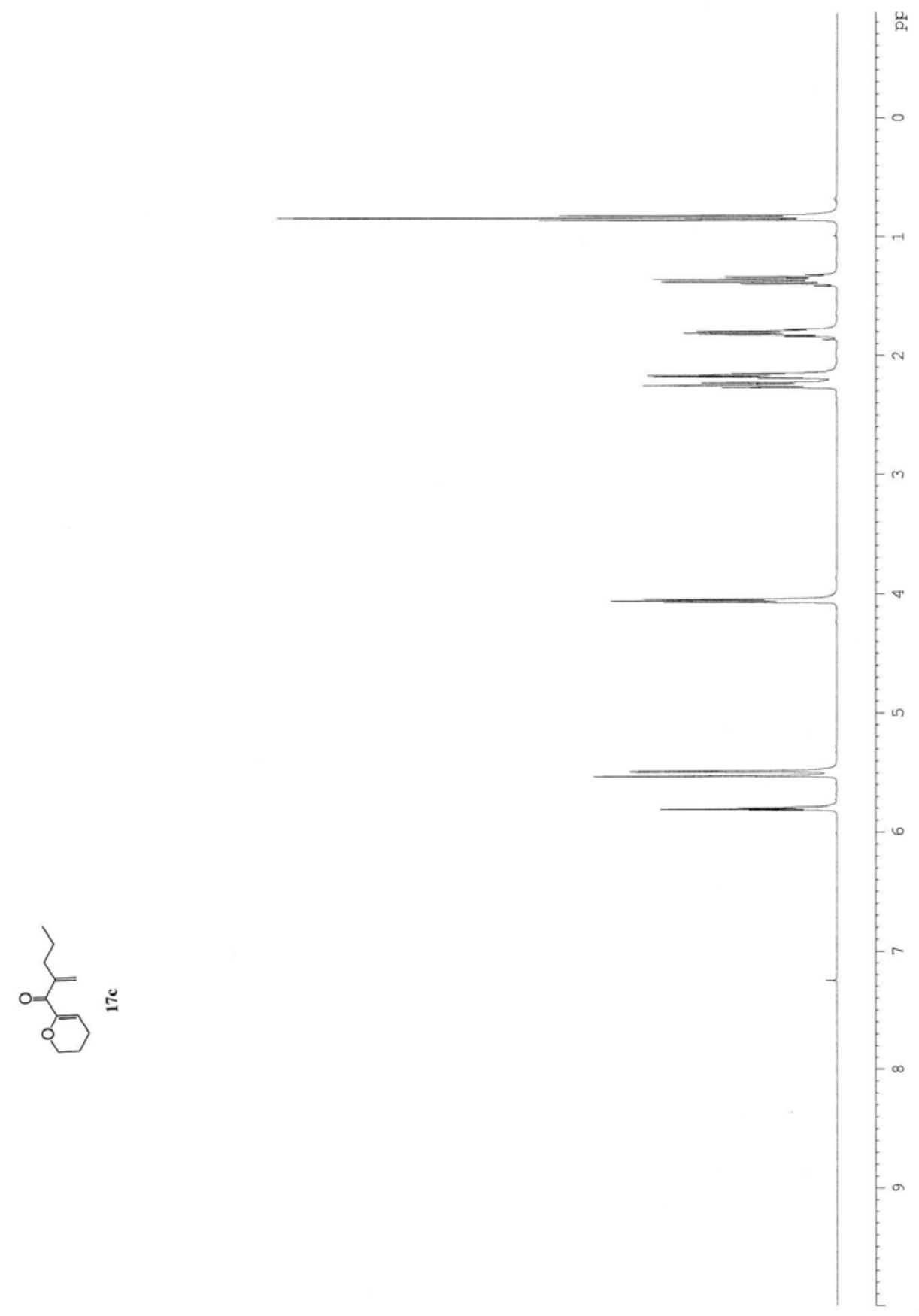

S12 


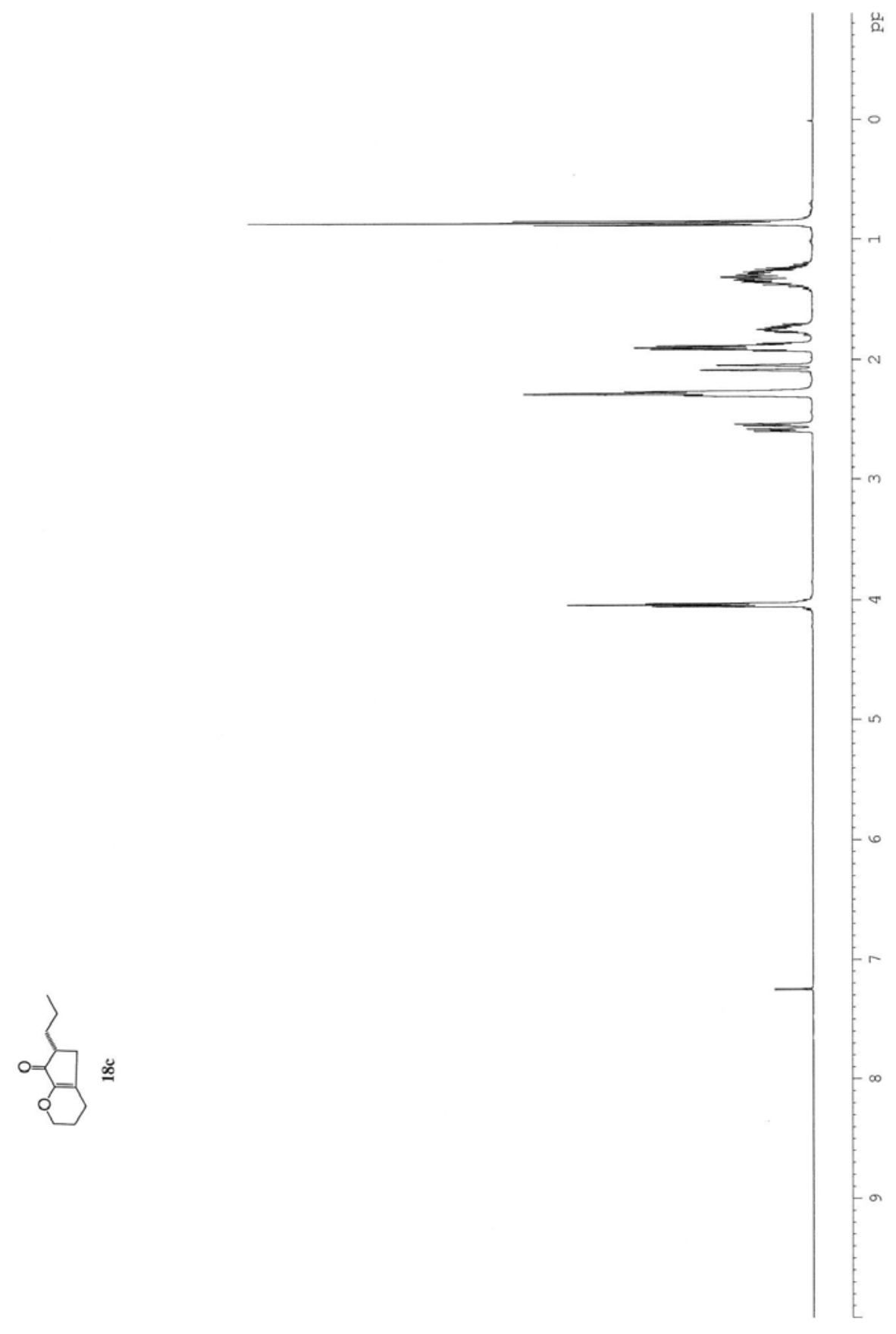

S13 


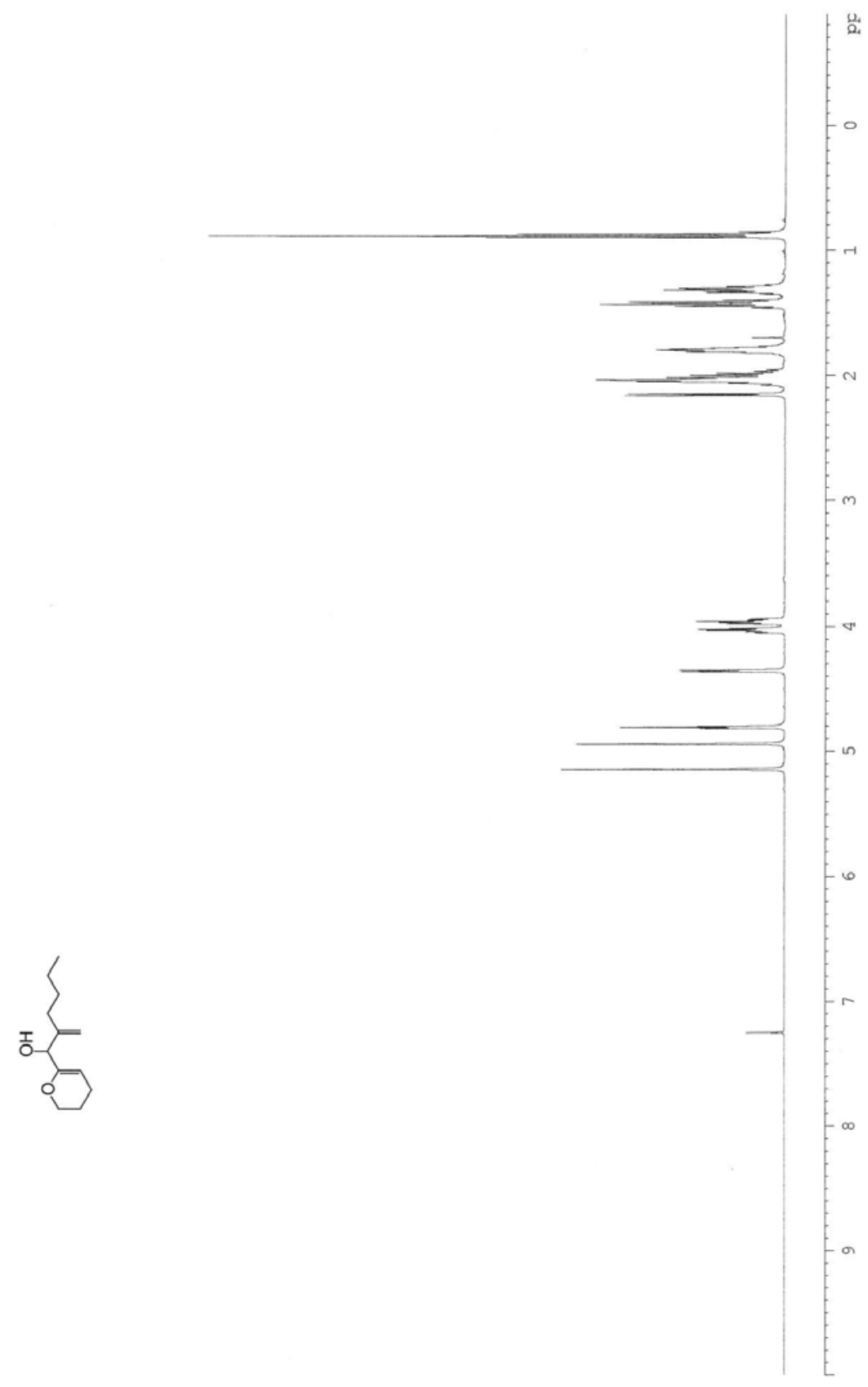




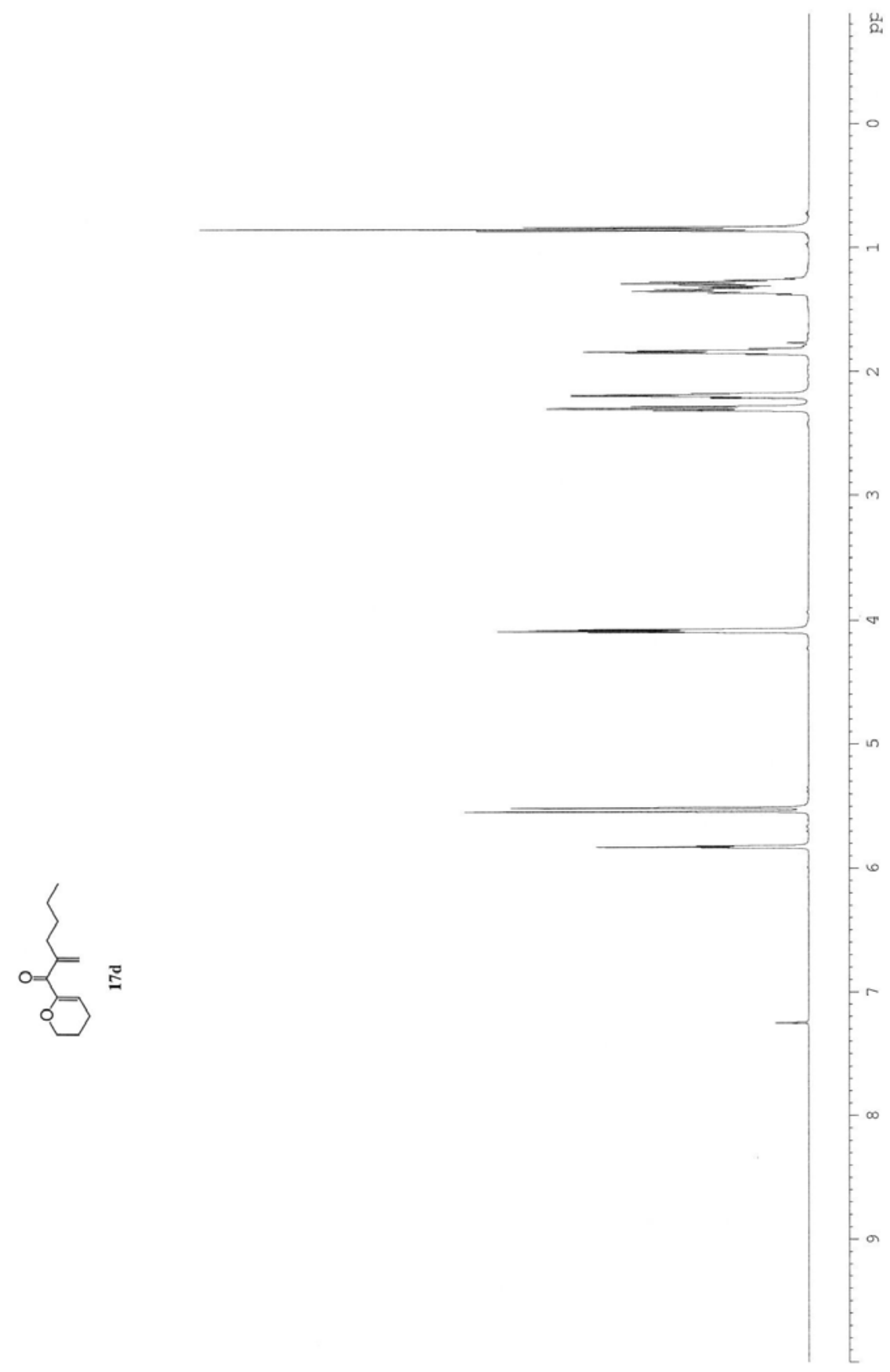

S15 


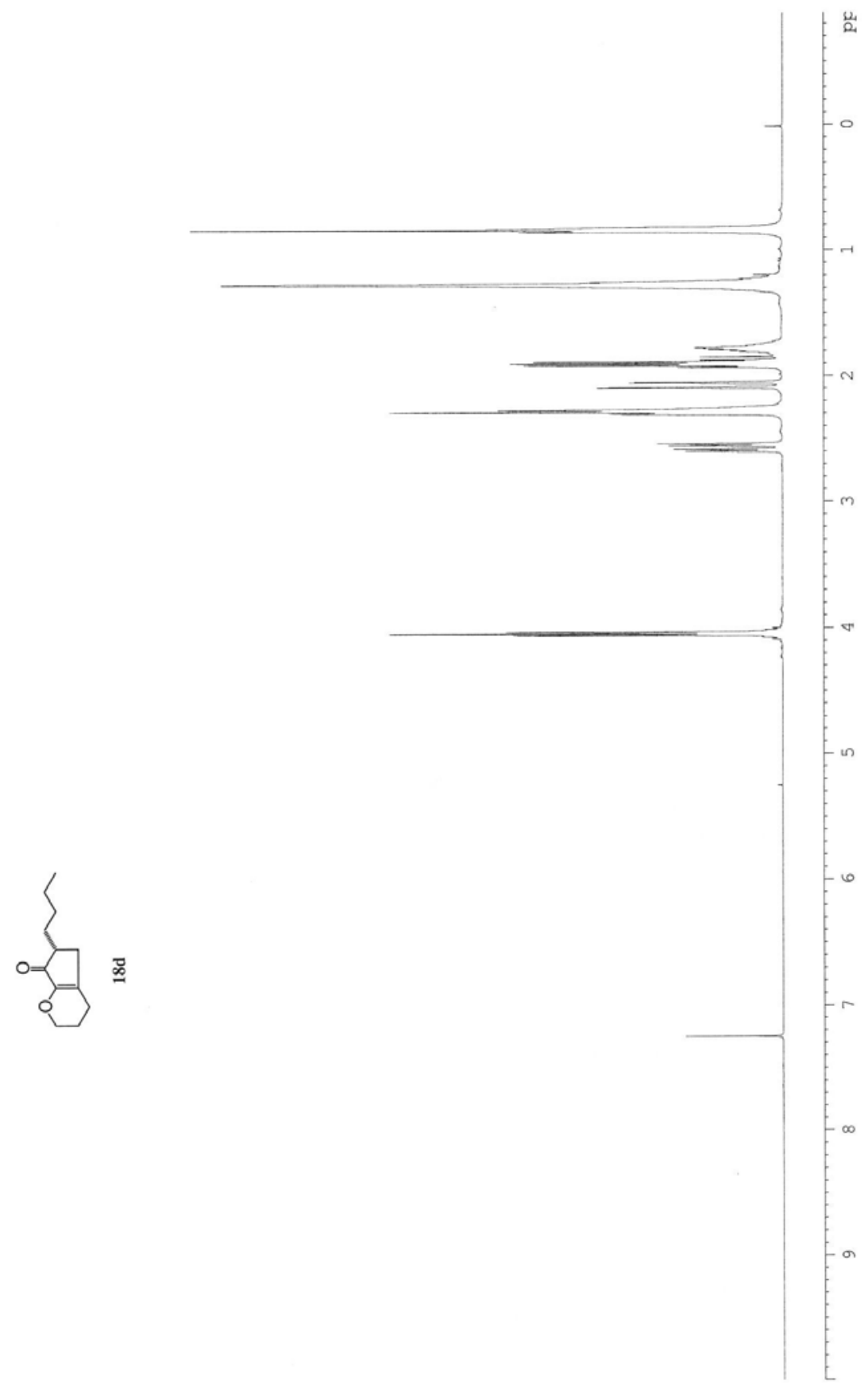

S16 


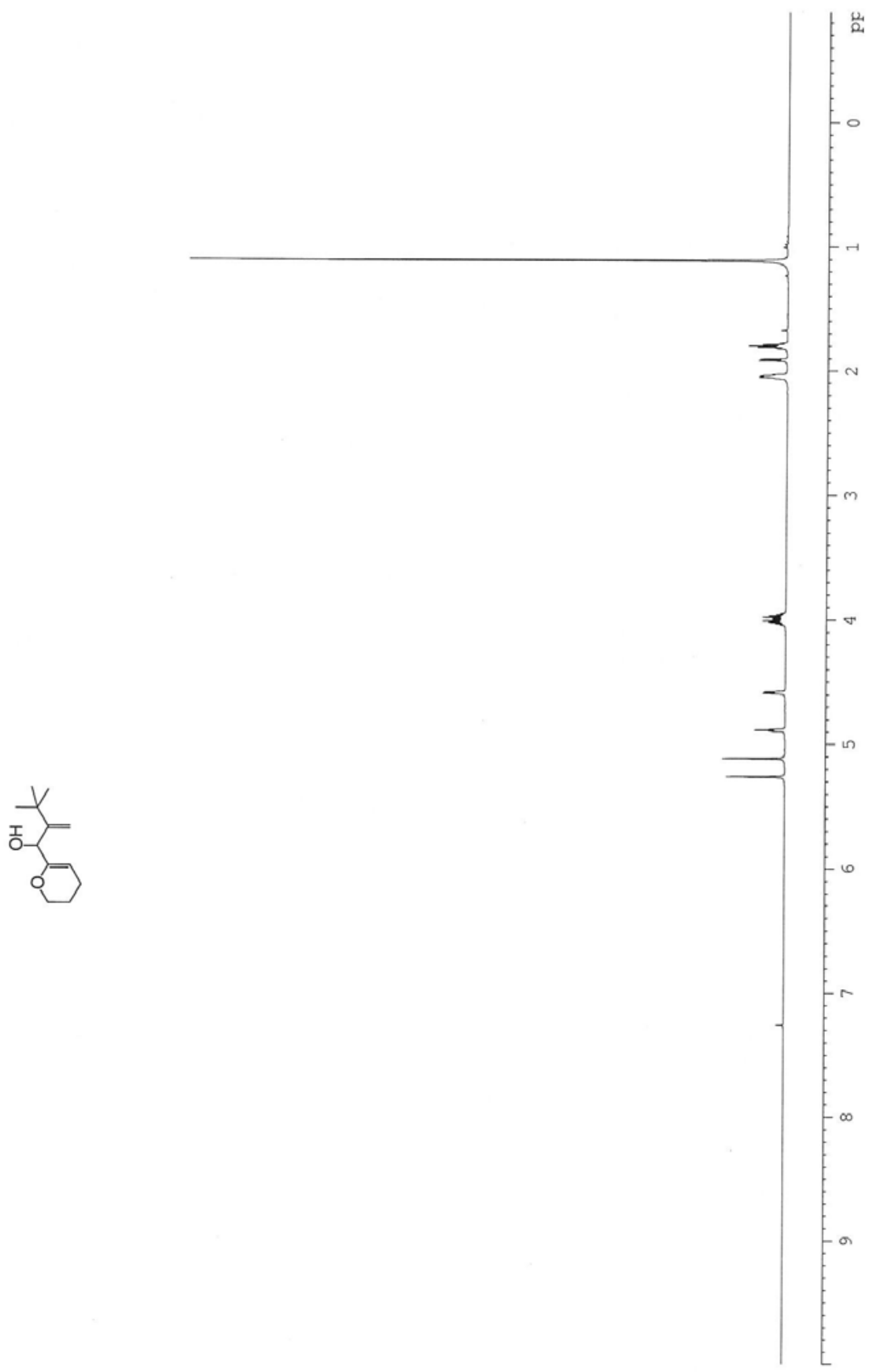




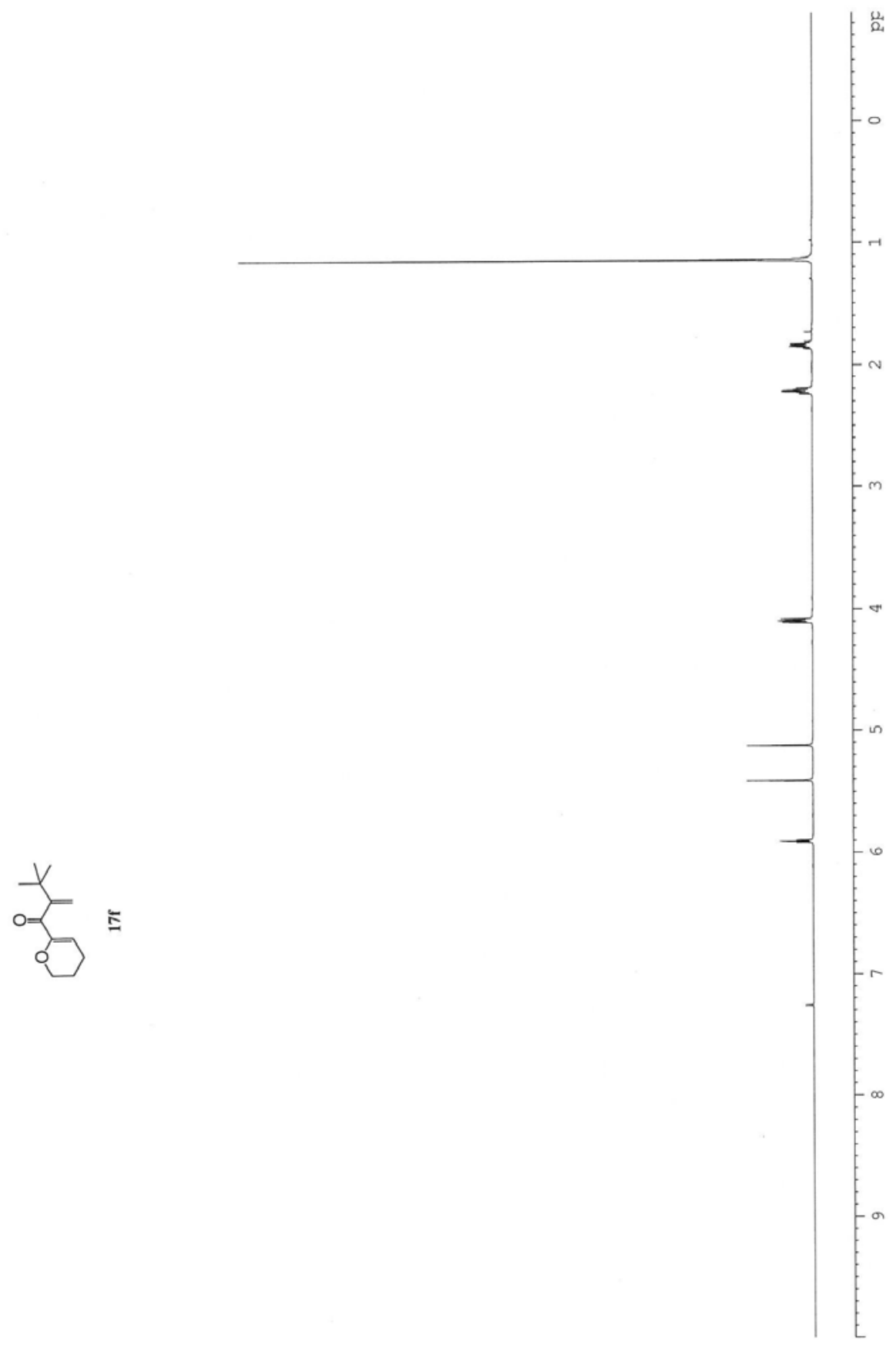




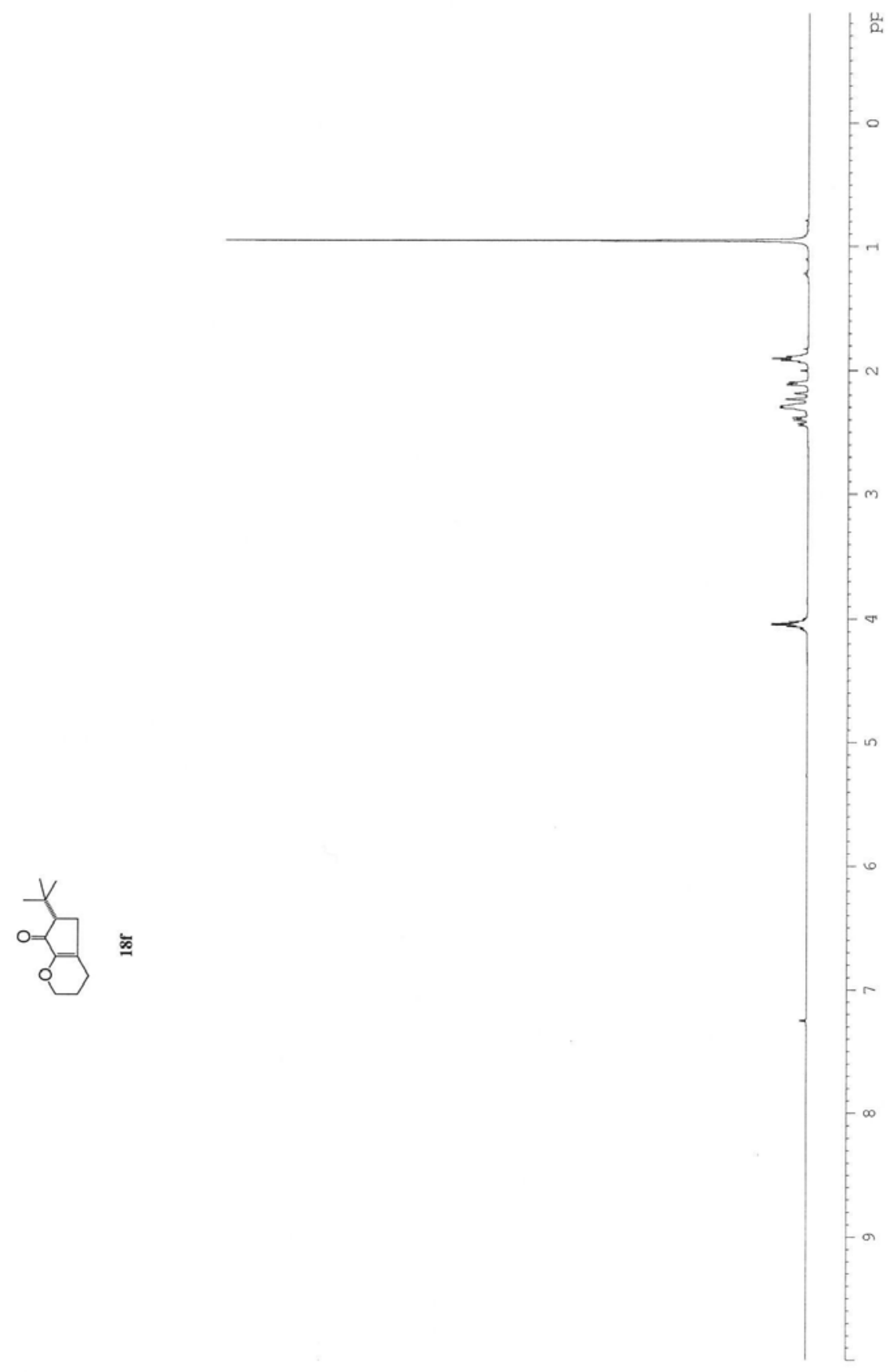




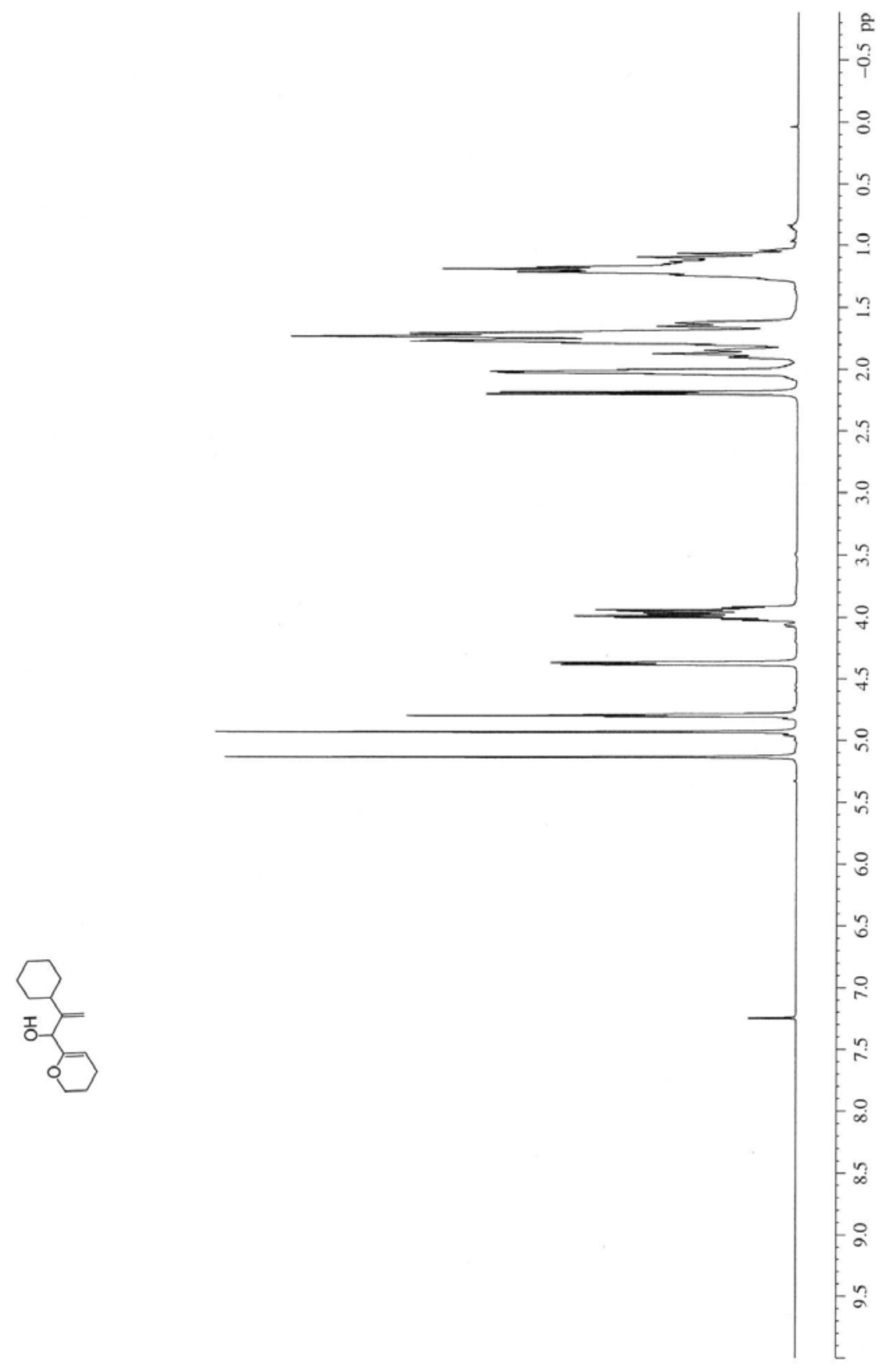




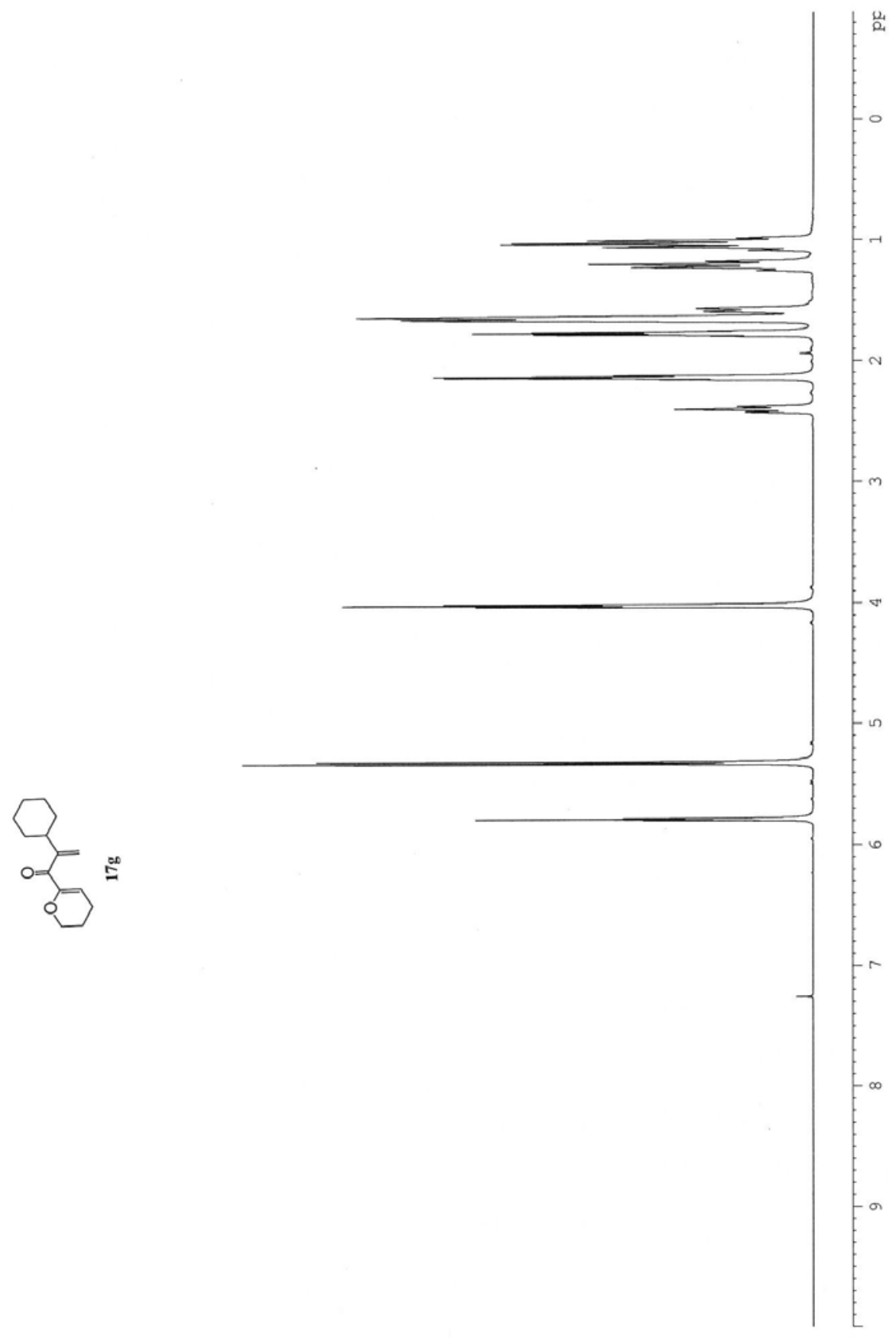




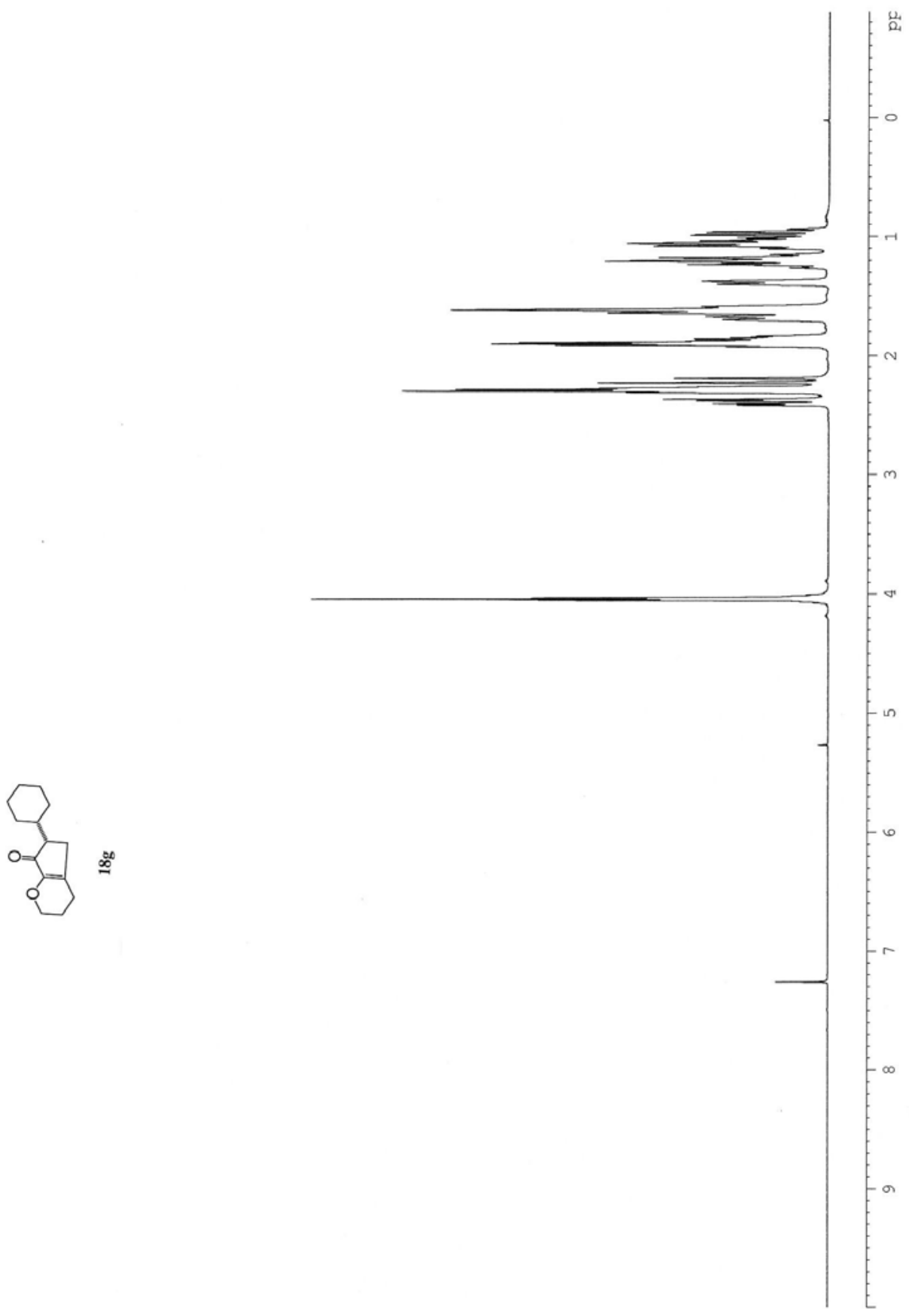




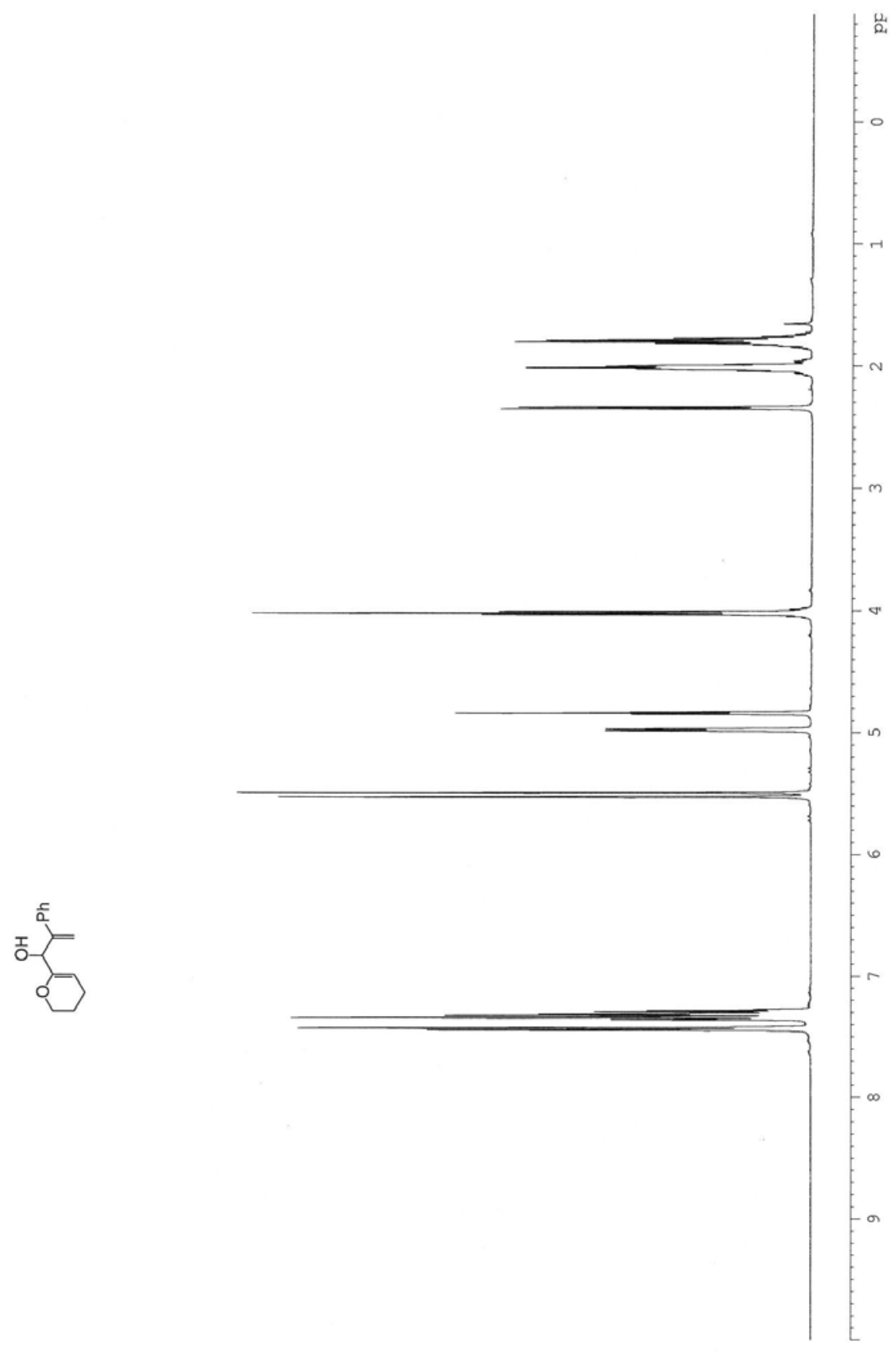




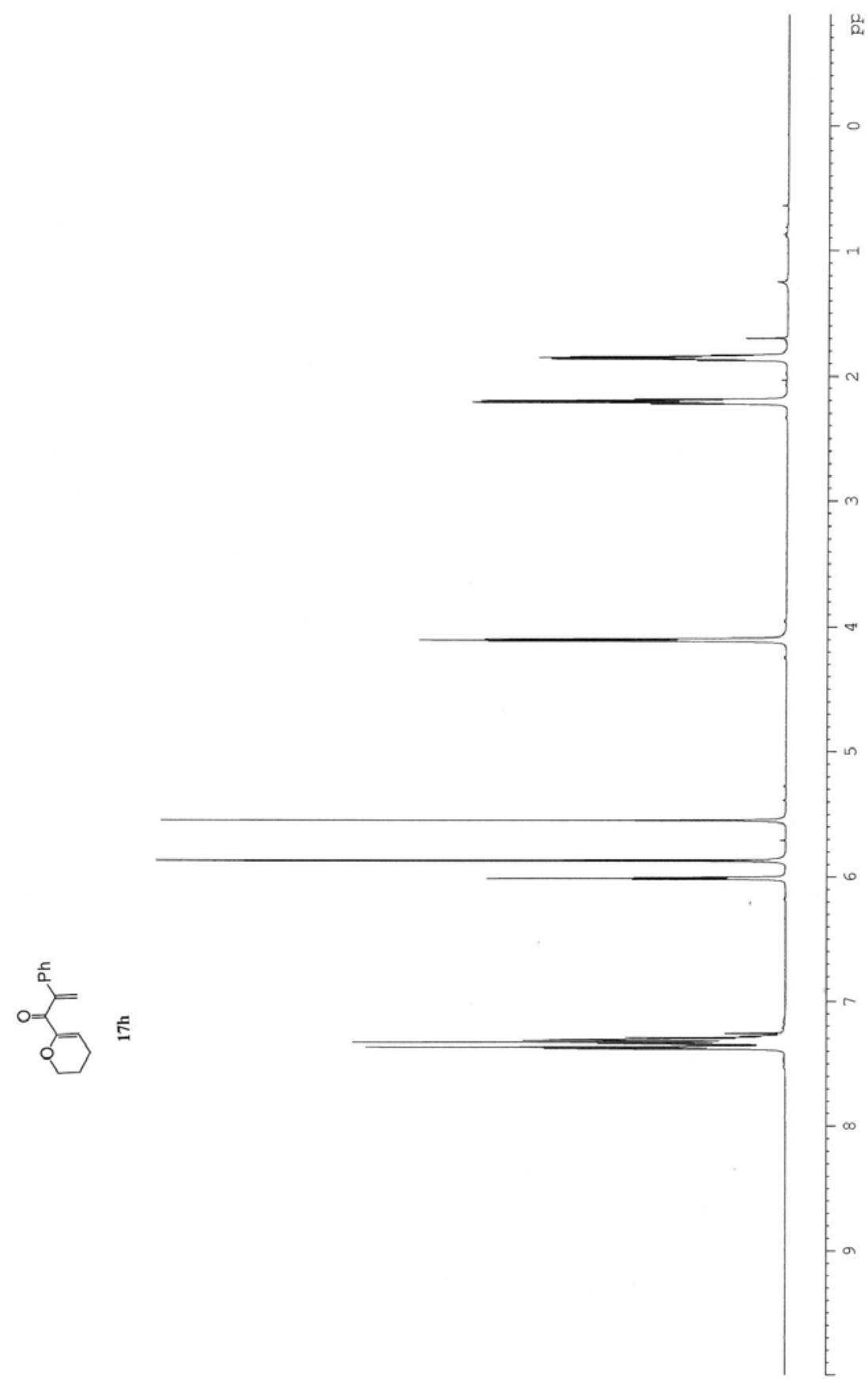




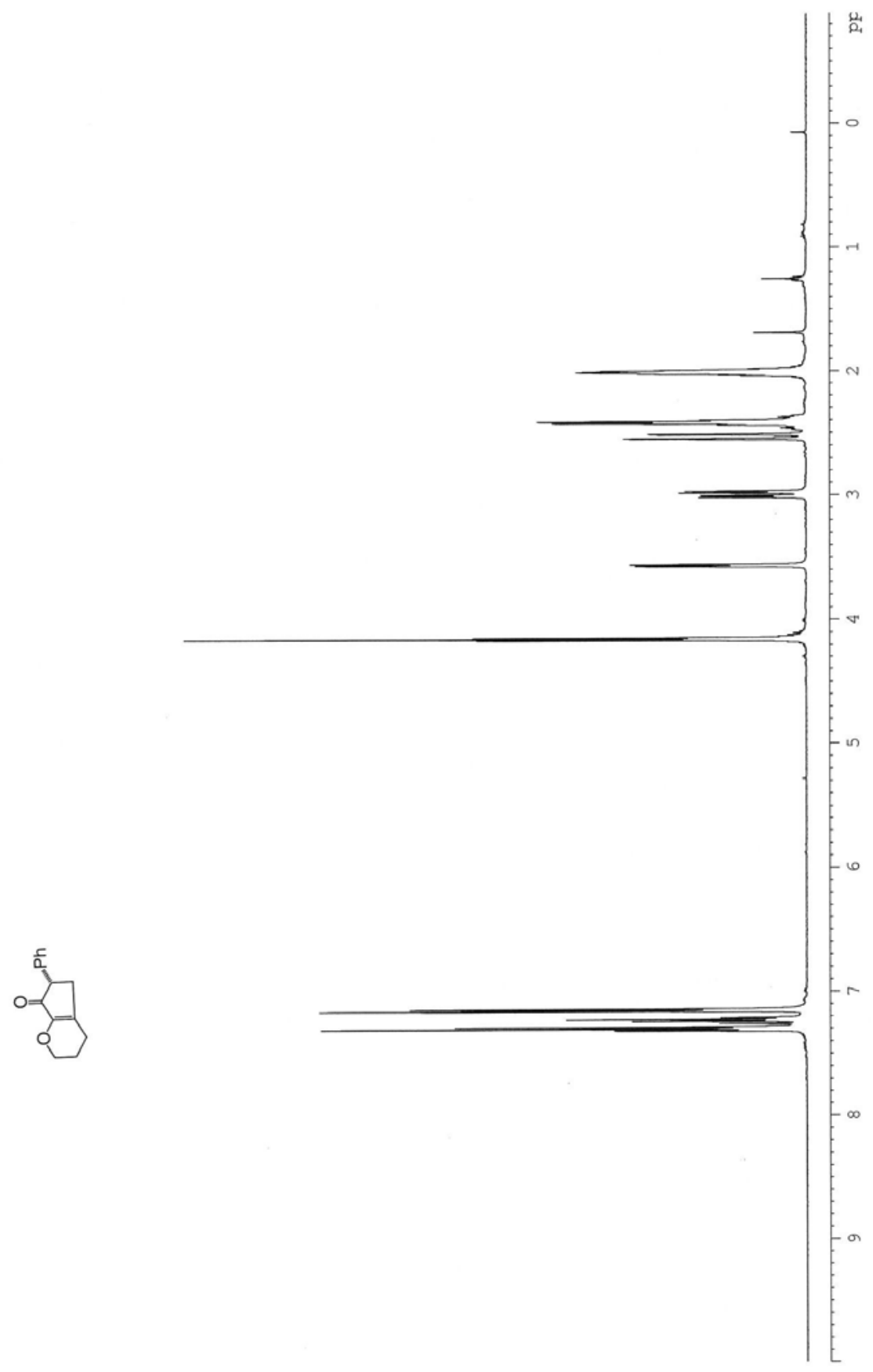




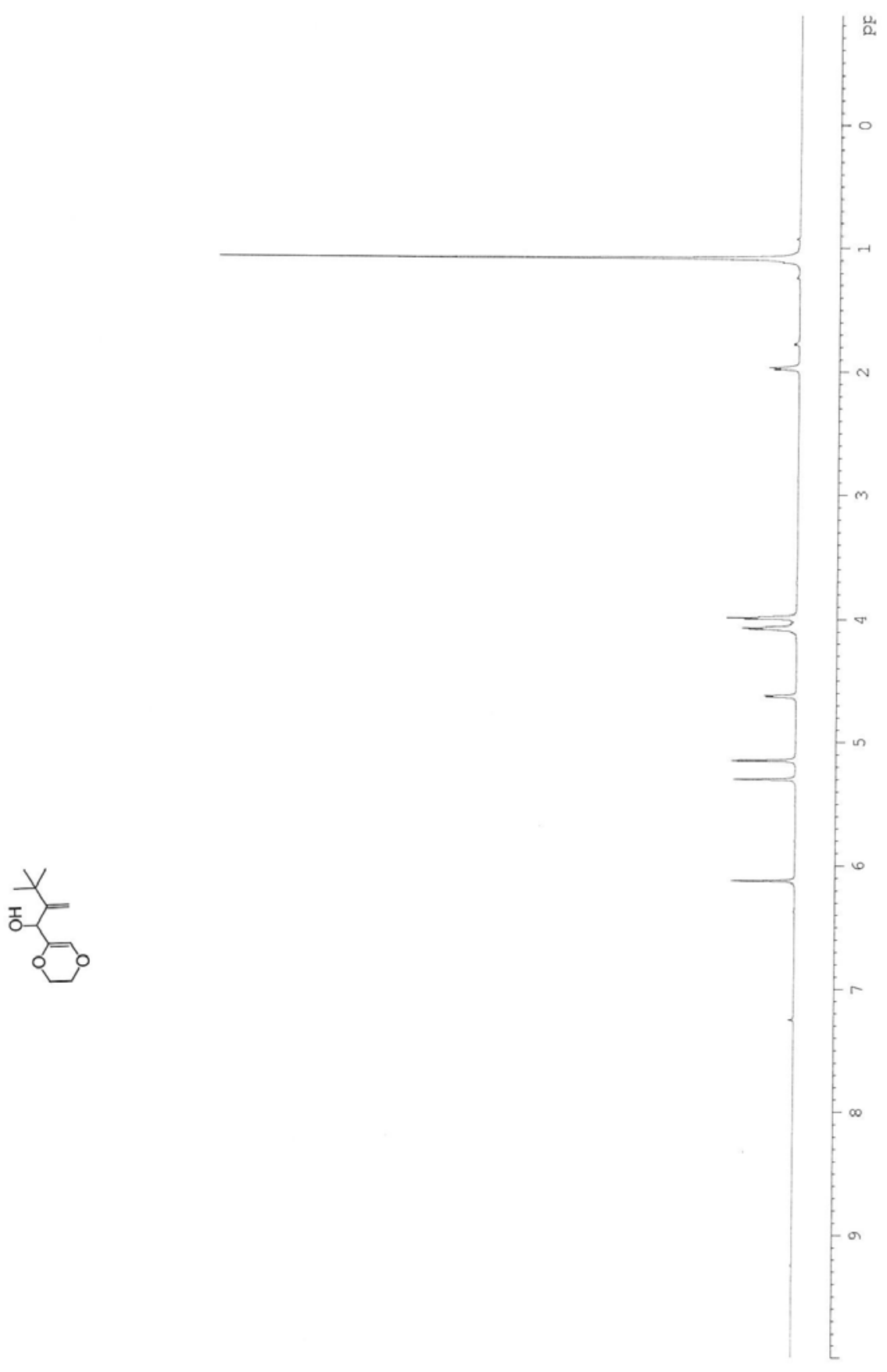




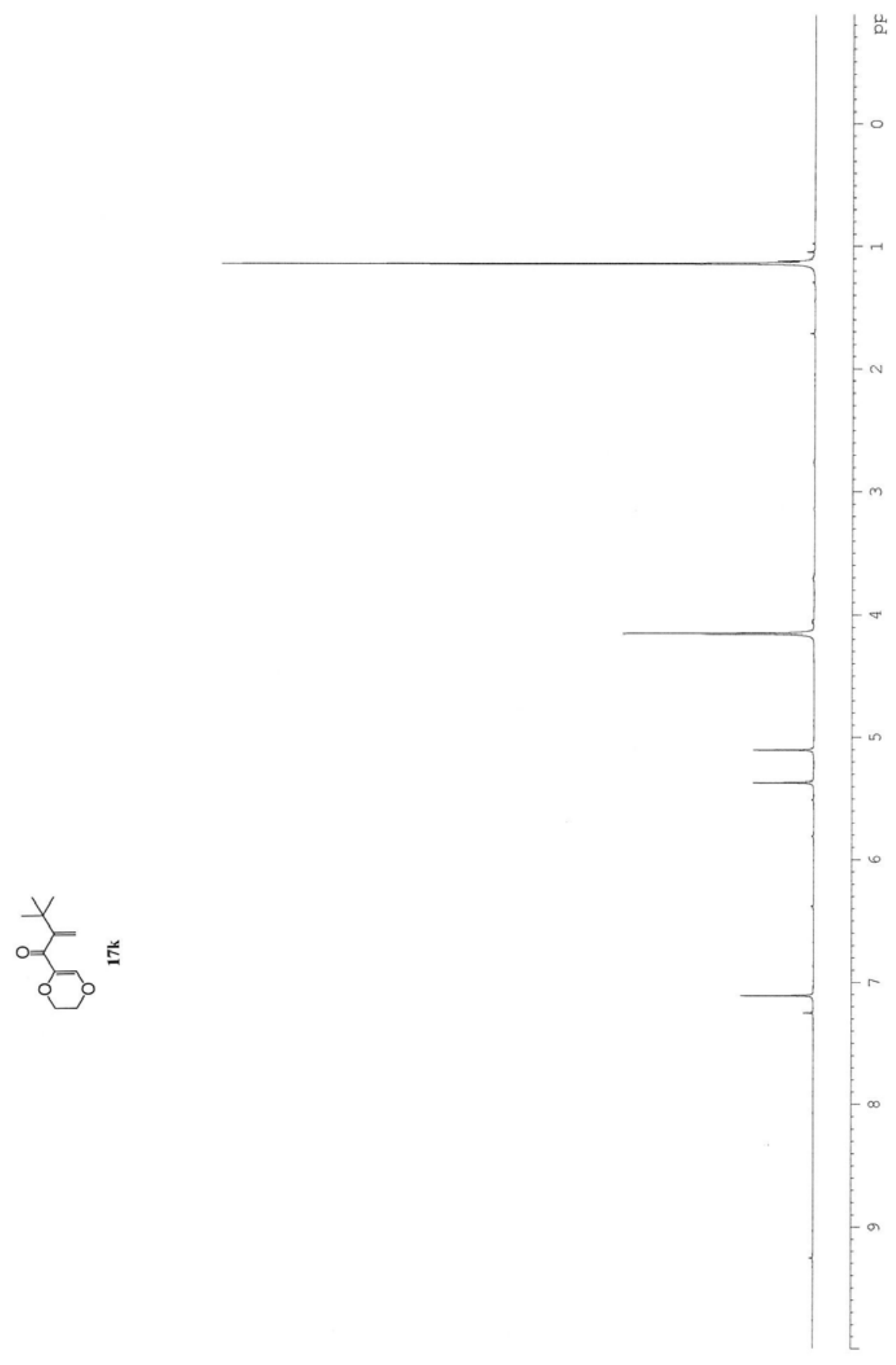




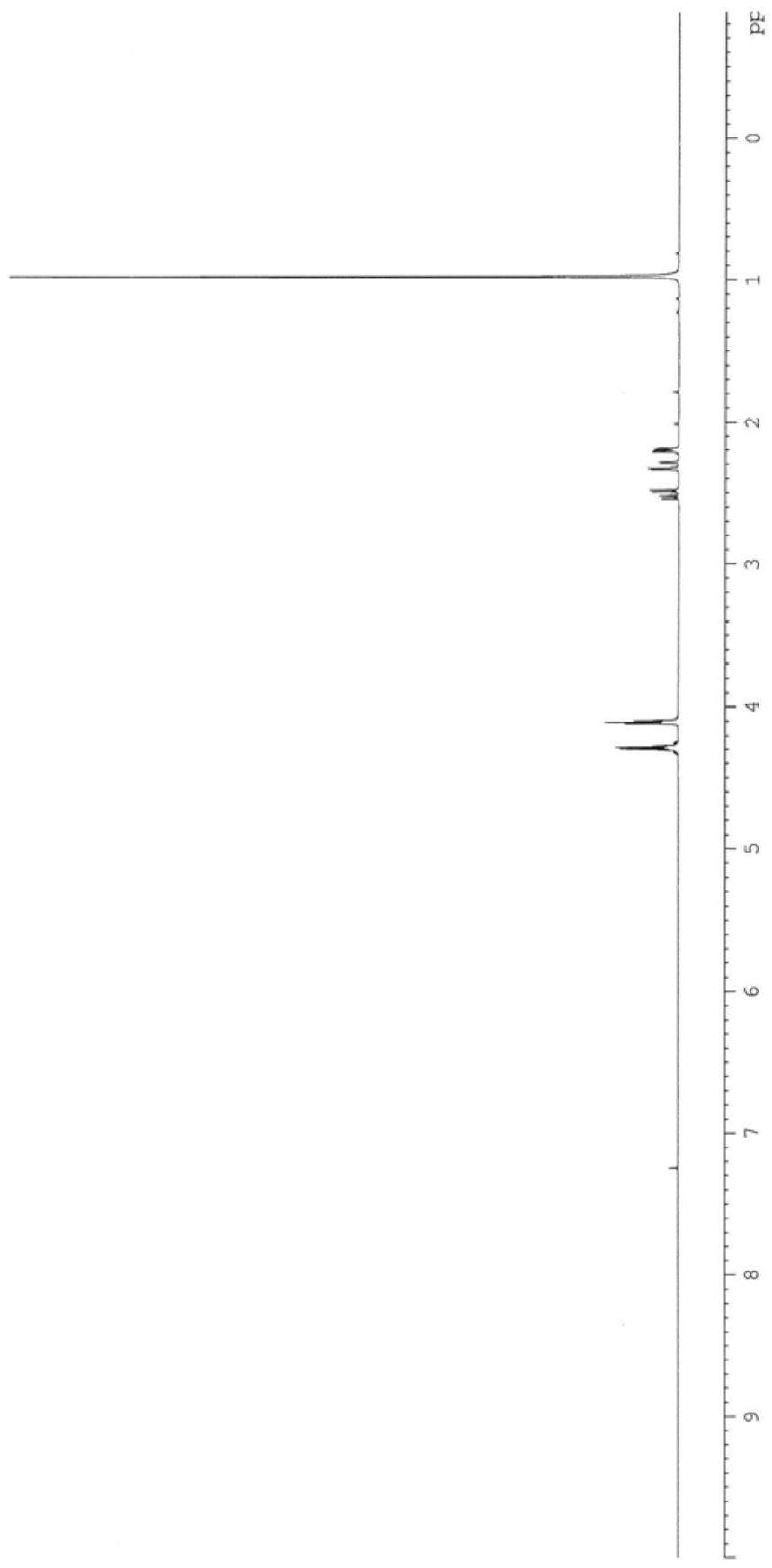

$=$ 


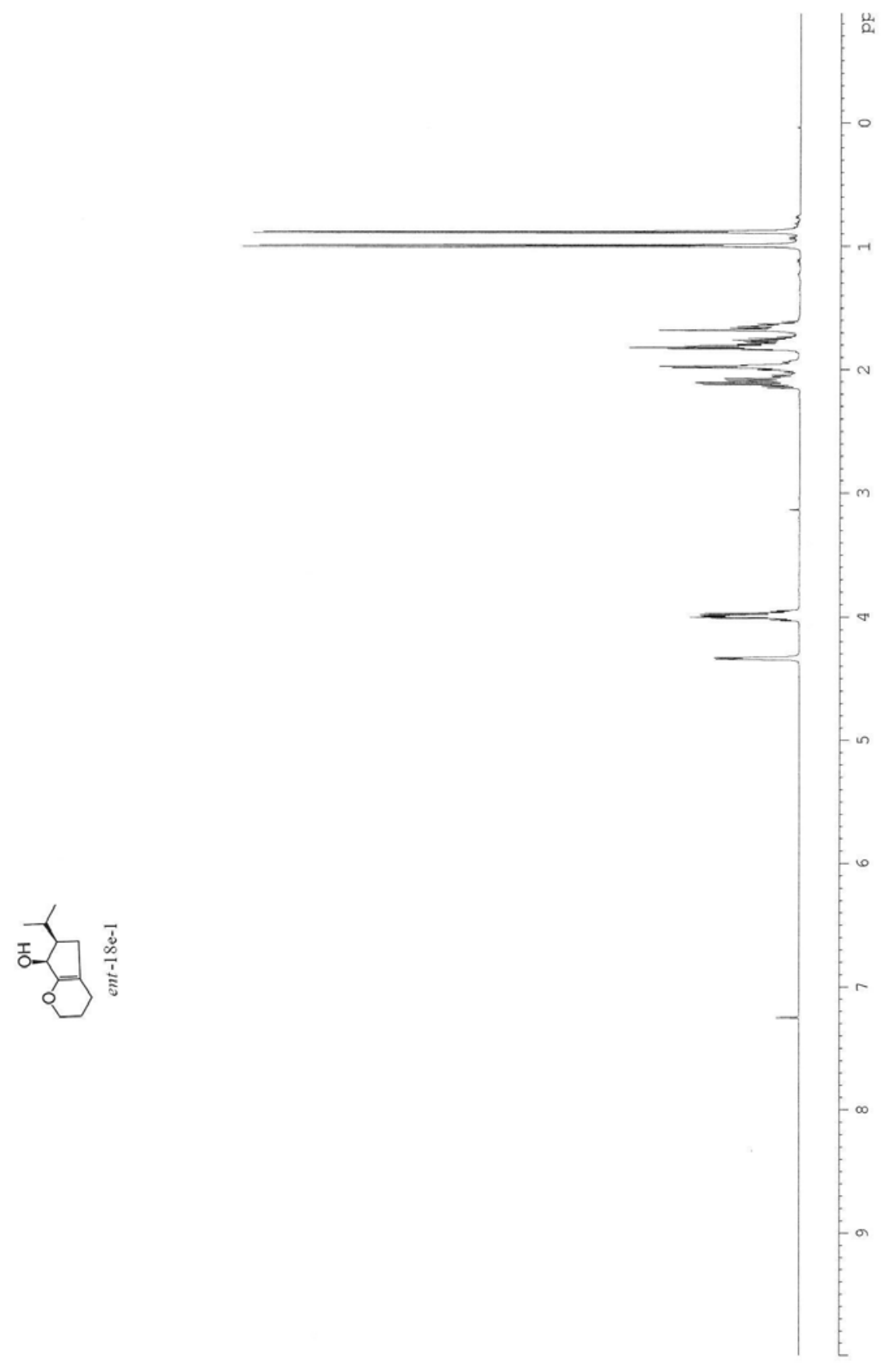




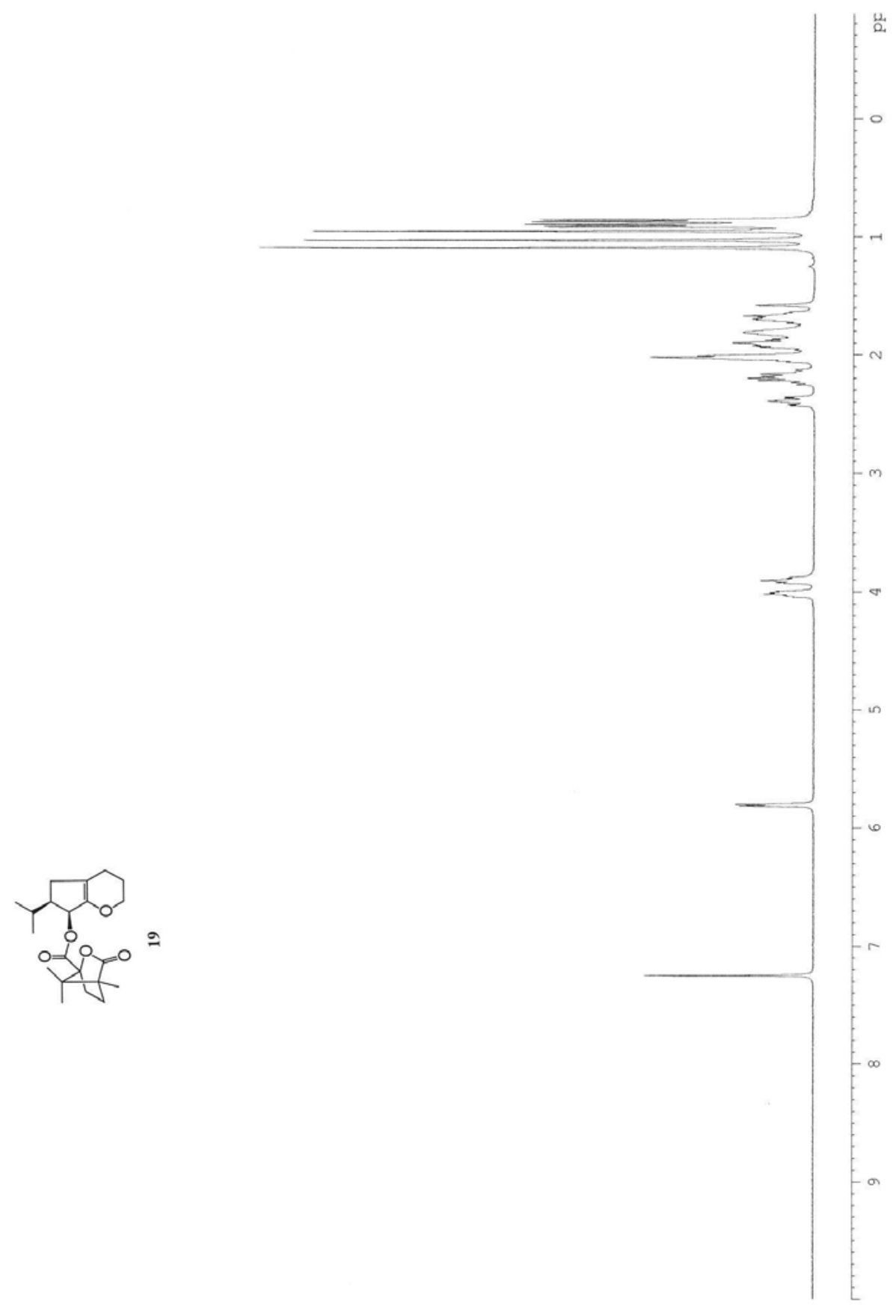


Data File C: \HPCHEM \I\DATA $\backslash$ G $\backslash$ NAZ00277.D
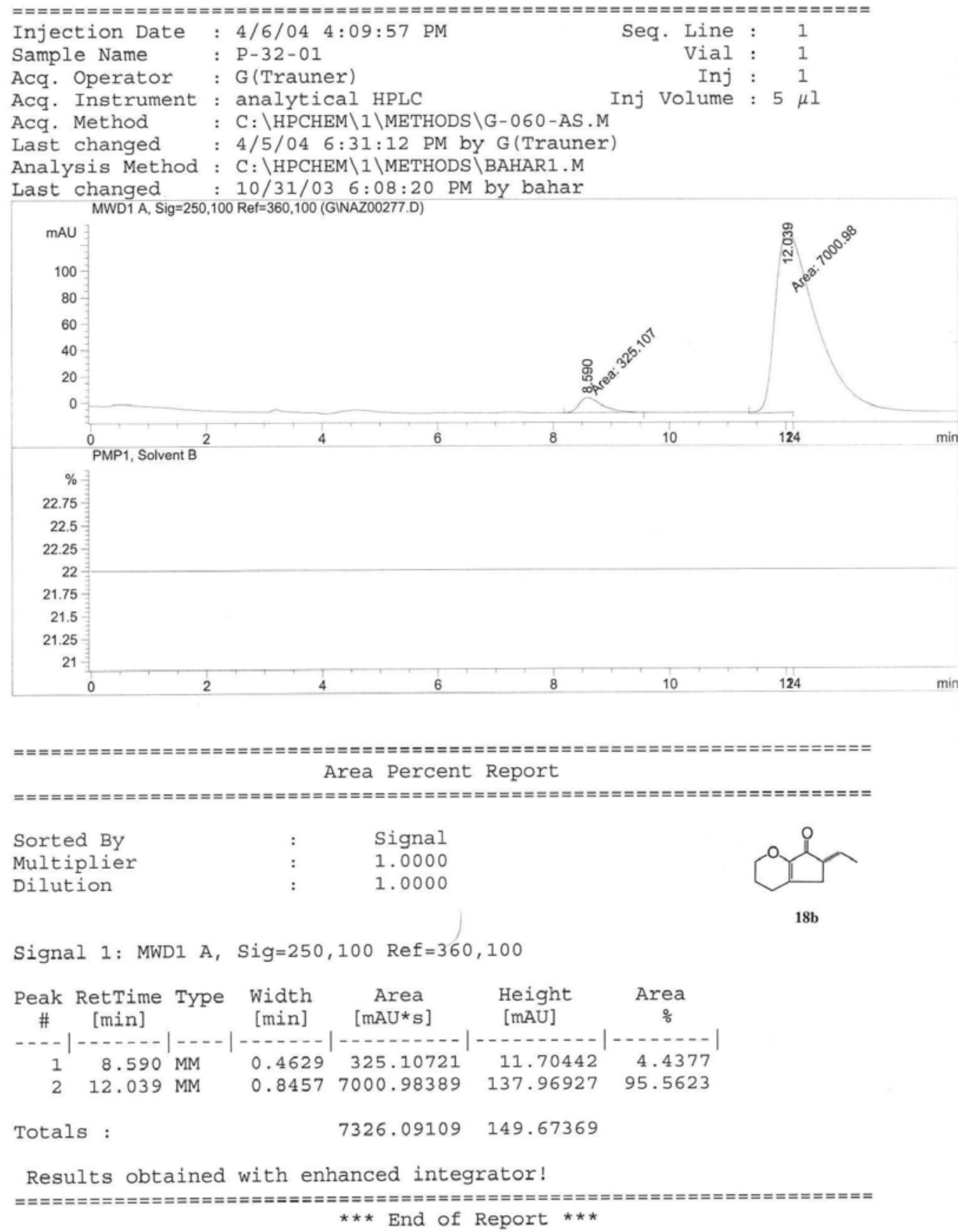

HP 1100 SYSTEM 4/20/04 5:27:40 PM bahar

Page 1 of 1 

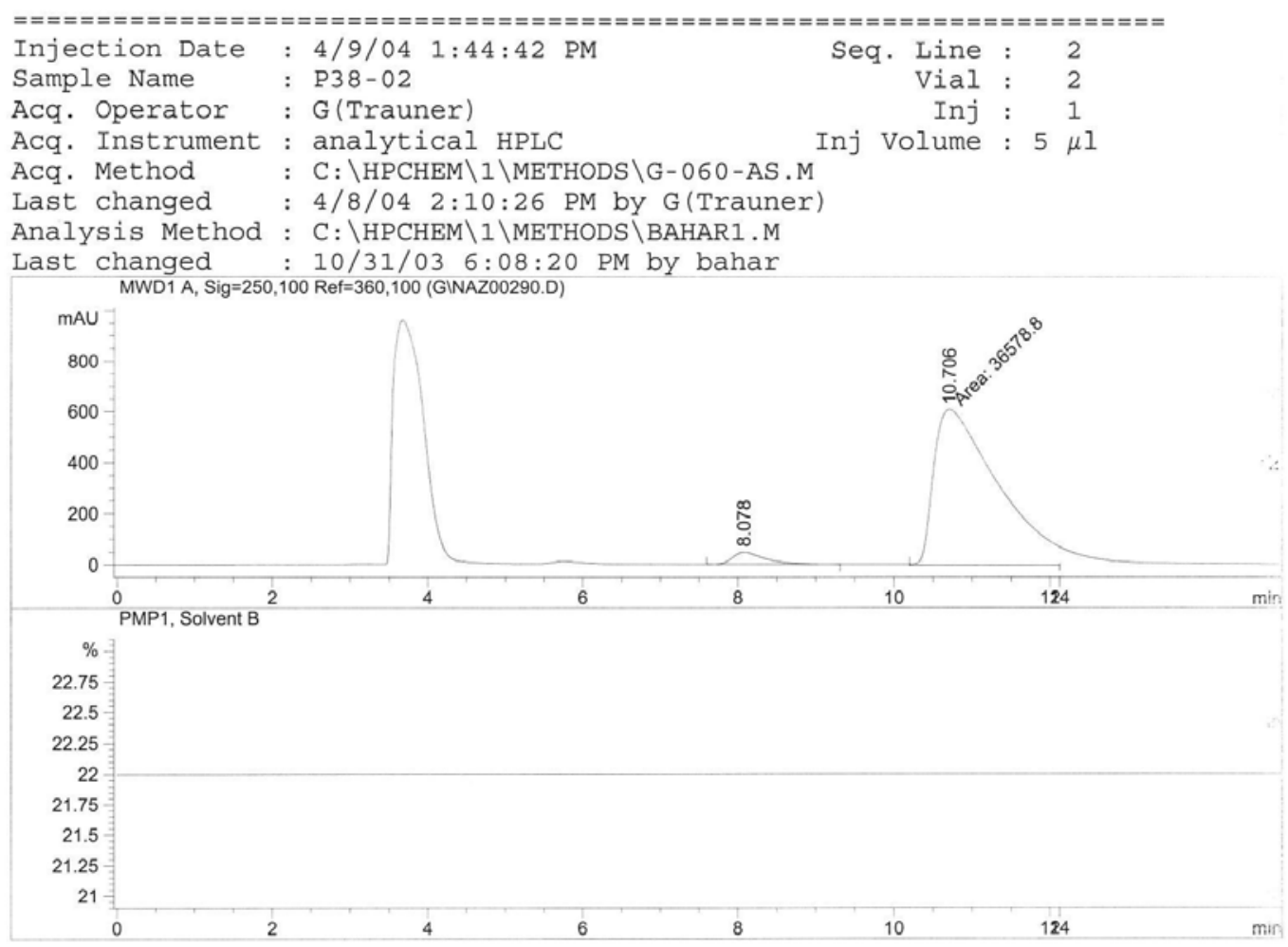

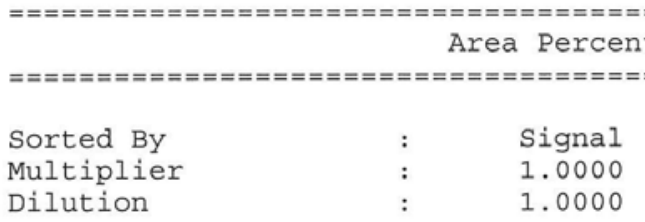

Signal 1: MWD1 A, Sig $=250,100$ Ref $=360,100$

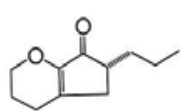

$18 \mathrm{c}$

\begin{tabular}{|c|c|c|c|c|c|c|}
\hline eak & $\begin{array}{l}\text { RetTime } \\
\text { [min] }\end{array}$ & ype & $\begin{array}{l}\text { Width } \\
\text { [min] }\end{array}$ & $\begin{array}{c}\text { Area } \\
{\left[\mathrm{mAU}^{*} \mathrm{~s}\right]}\end{array}$ & $\begin{array}{l}\text { Height } \\
\text { [mAU] }\end{array}$ & $\begin{array}{c}\text { Area } \\
\frac{\%}{6}\end{array}$ \\
\hline & & & & & & \\
\hline & & & & & $\begin{array}{r}47.02840 \\
611.70398\end{array}$ & 96.485 \\
\hline
\end{tabular}

Totals : $\quad 3.79112 \mathrm{e} 4 \quad 658.73238$

Results obtained with enhanced integrator!

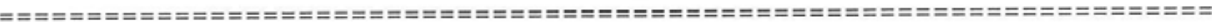

*** End of Report ***

HP 1100 SYSTEM 4/20/04 5:28:36 PM bahar

Page 1 of 1 

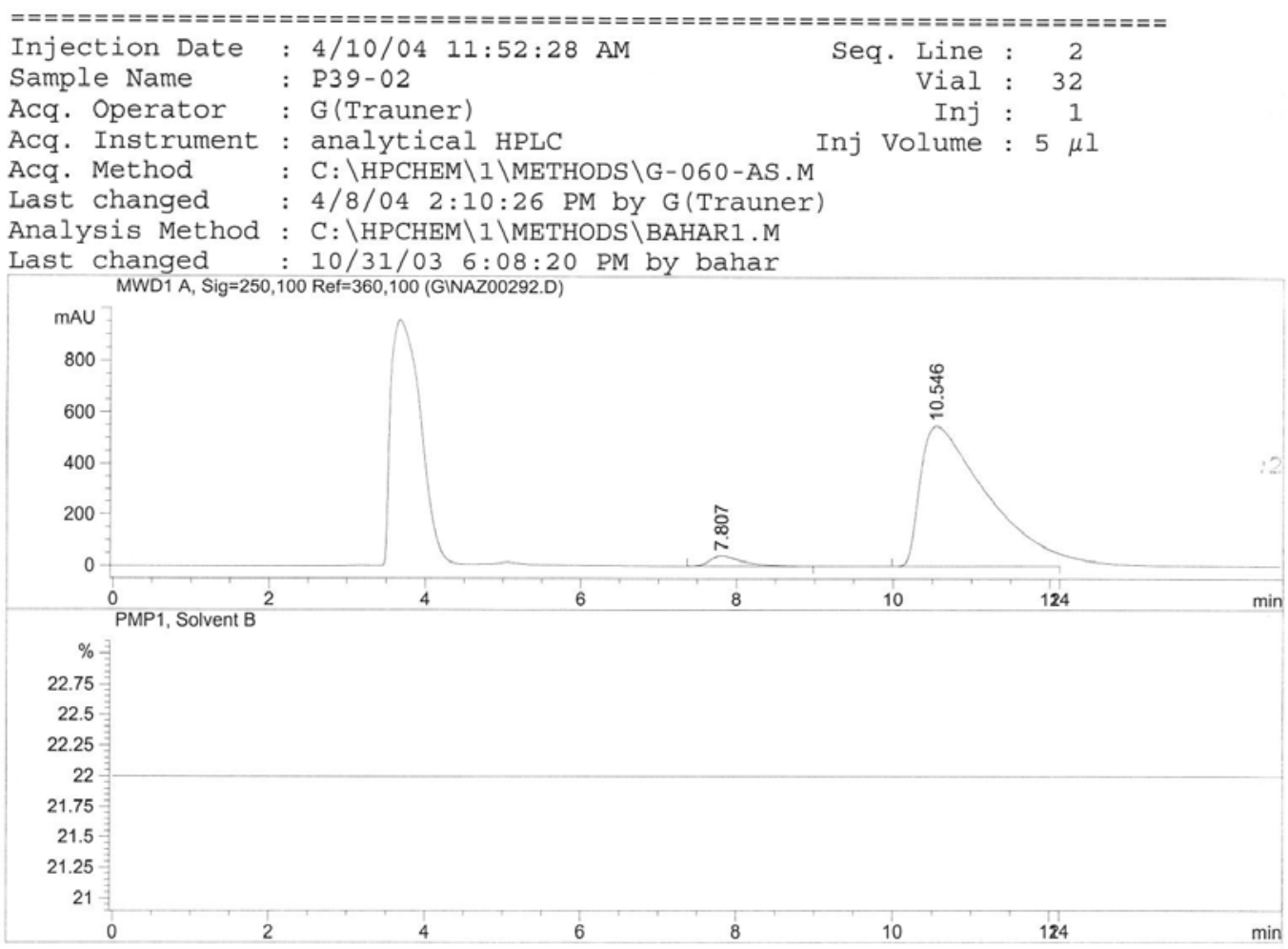

\begin{tabular}{|c|c|c|}
\hline Sorted By & : & Signal \\
\hline Multiplier & : & 1.0000 \\
\hline Dilution & : & 1.0000 \\
\hline
\end{tabular}

Signal 1: MWD1 A, Sig $=250,100$ Ref $=360,100$

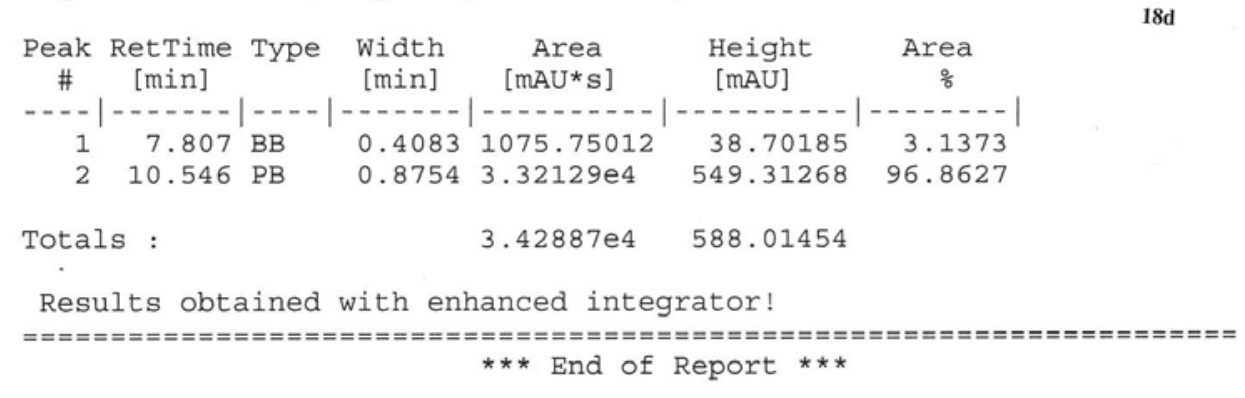




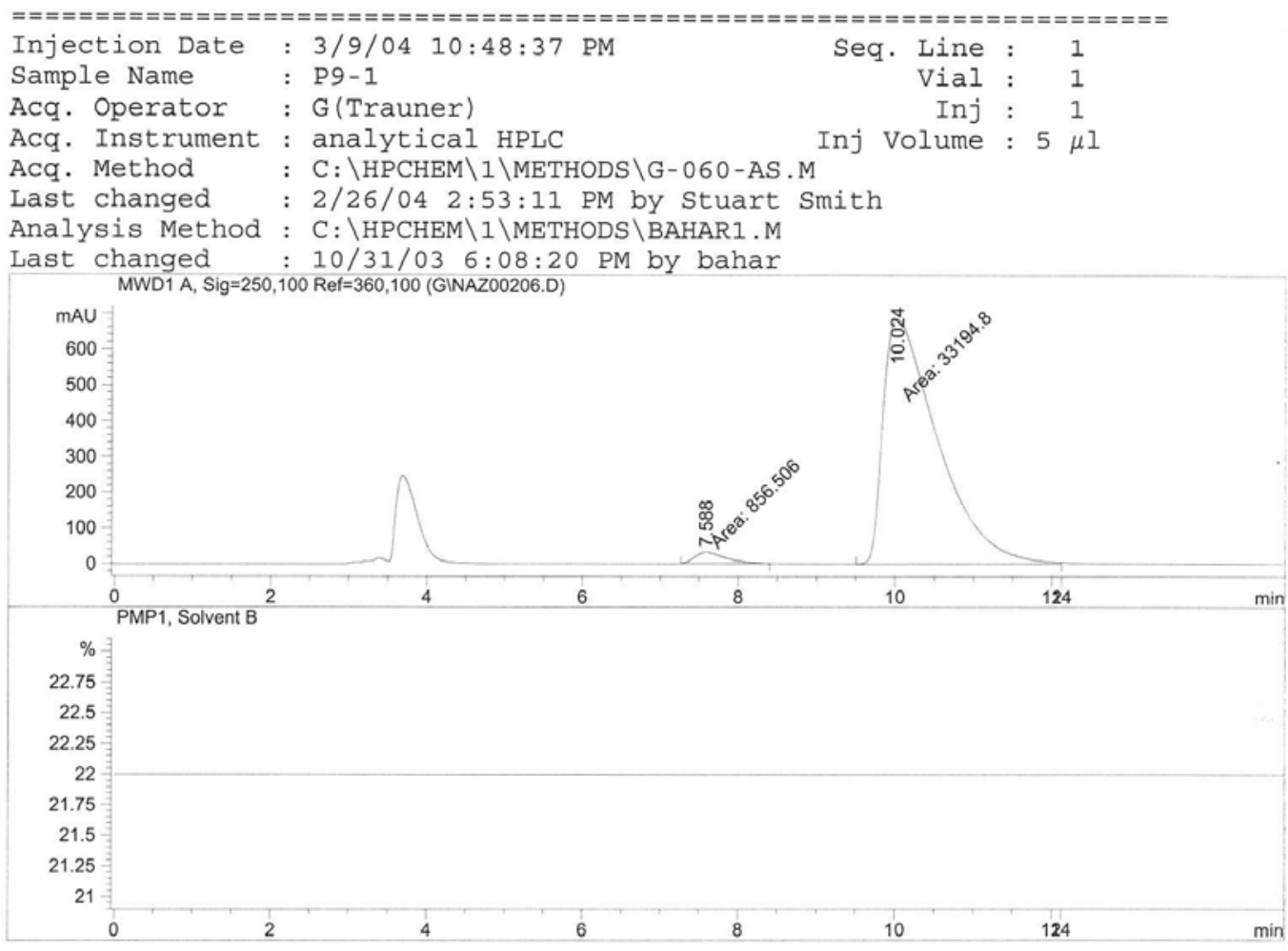

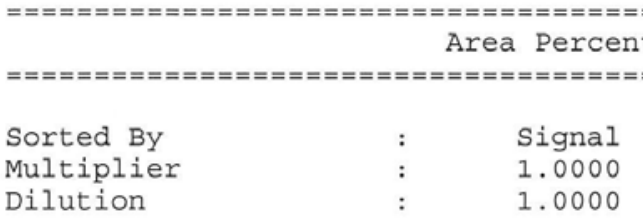

Signal 1: MWD1 A, Sig=250,100 Ref=360,100

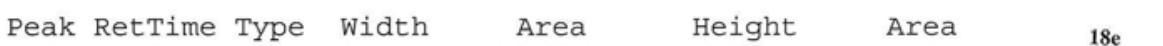

\begin{tabular}{|c|c|c|c|c|c|c|}
\hline \# & [min] & Tyре & $\begin{array}{l}\text { Width } \\
\text { [min] }\end{array}$ & $\begin{array}{c}\text { Area } \\
{\left[\mathrm{mAU}{ }^{*} \mathrm{~s}\right]}\end{array}$ & $\begin{array}{l}\text { Height } \\
\text { [mAU] }\end{array}$ & $\begin{array}{c}\text { Area } \\
\text { \% }\end{array}$ \\
\hline & & & & - & -- & - \\
\hline 1 & 7.588 & MM & 0.4515 & 856.50562 & 31.61518 & 2.515 \\
\hline 2 & 10.024 & MM & 0.8083 & 3. $31948 \mathrm{e} 4$ & 684.48767 & 97.4847 \\
\hline
\end{tabular}

$$
\text { Totals : } \quad 3.40513 e 4 \quad 716.10285
$$

Results obtained with enhanced integrator!

$\star * \star$ End of Report $* * *$ 


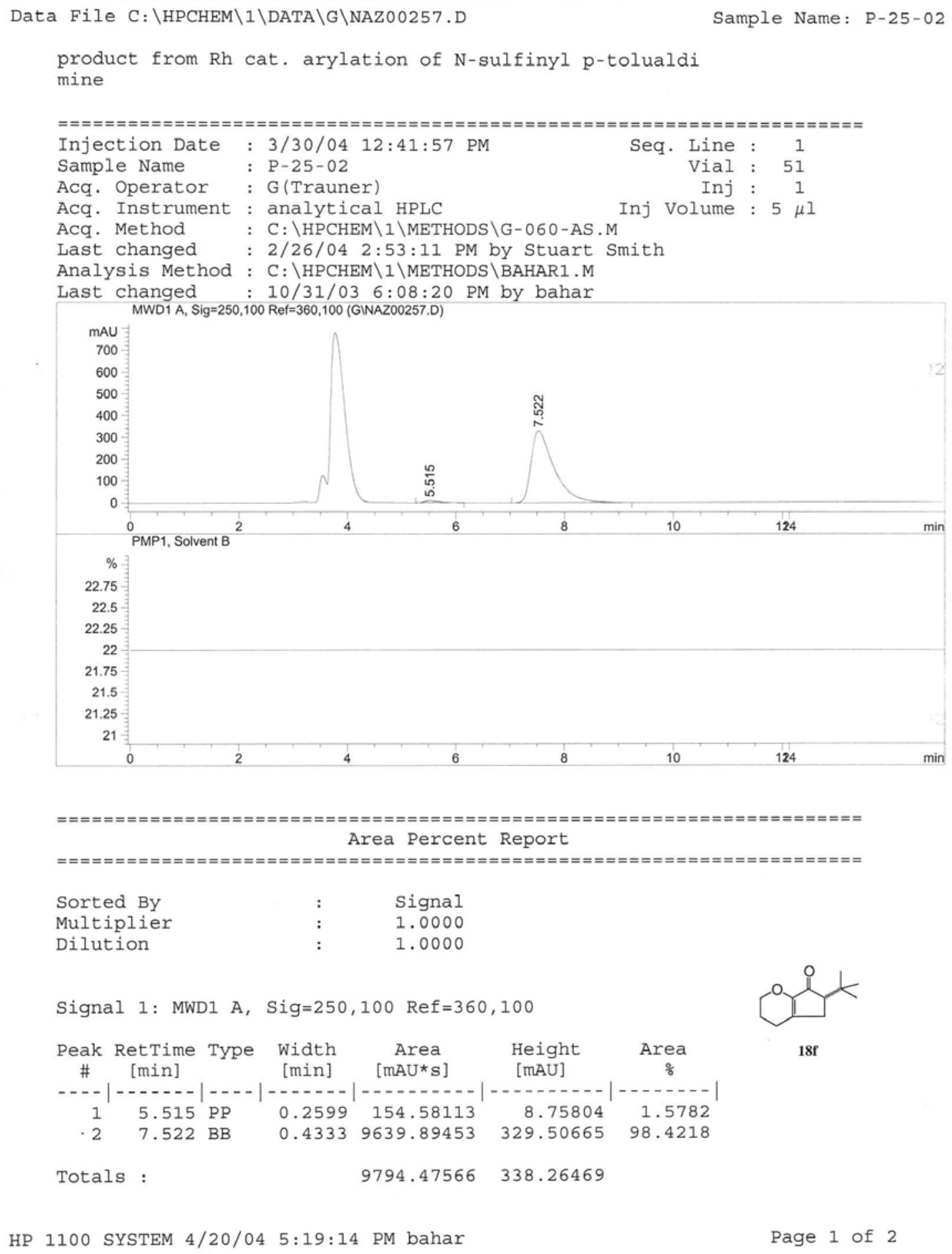




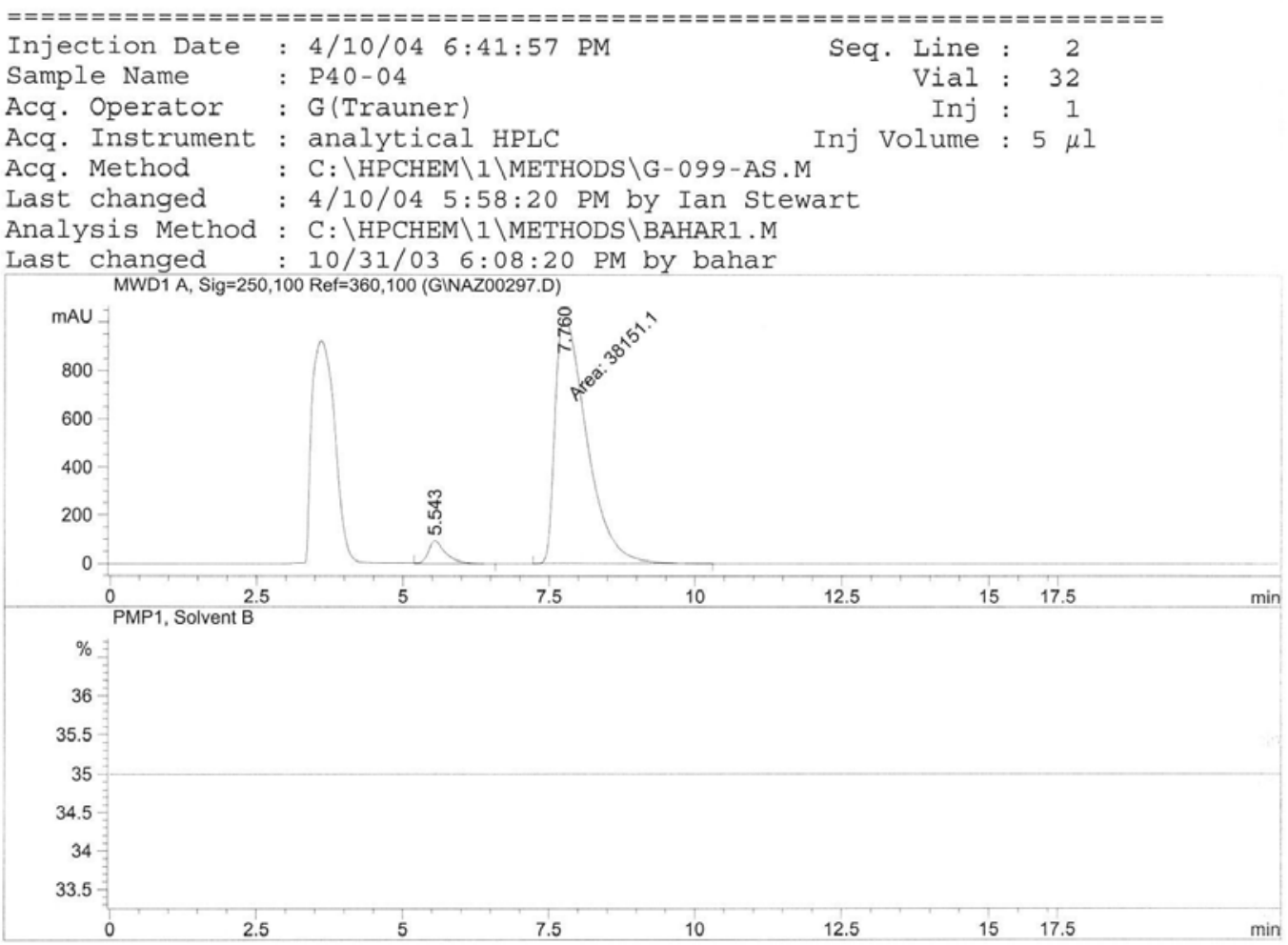

\begin{tabular}{|c|c|c|}
\hline Sorted By & : & Signa \\
\hline Multiplier & : & 1.0000 \\
\hline Dilution & : & 1.0000 \\
\hline
\end{tabular}

Signal 1: MWD1 A, Sig $=250,100$ Ref $=360,100$

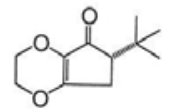

$18 \mathrm{k}$

\begin{tabular}{|c|c|c|c|c|c|c|}
\hline $\begin{array}{c}\text { Peak } \\
\#\end{array}$ & $\begin{array}{c}\text { RetTime } \\
\text { [min] }\end{array}$ & Type & $\begin{array}{l}\text { Width } \\
\text { [min] }\end{array}$ & $\begin{array}{c}\text { Area } \\
{\left[\mathrm{mAU}{ }^{*} \mathrm{~s}\right]}\end{array}$ & $\begin{array}{l}\text { Height } \\
\text { [mAU] }\end{array}$ & $\begin{array}{c}\text { Area } \\
\%\end{array}$ \\
\hline & & & & ---- & -- & ------- \\
\hline 1 & 5.543 & BB & 0.2982 & 1899.12390 & 93.01701 & 4.7419 \\
\hline 2 & 7.760 & MM & 0.6264 & $3.81511 \mathrm{e} 4$ & 1015.16644 & 95.2581 \\
\hline
\end{tabular}

Totals :

$4.00502 \mathrm{e} 4 \quad 1108.18345$

Results obtained with enhanced integrator!

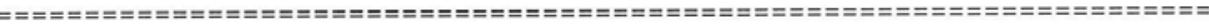

*** End of Report *** 

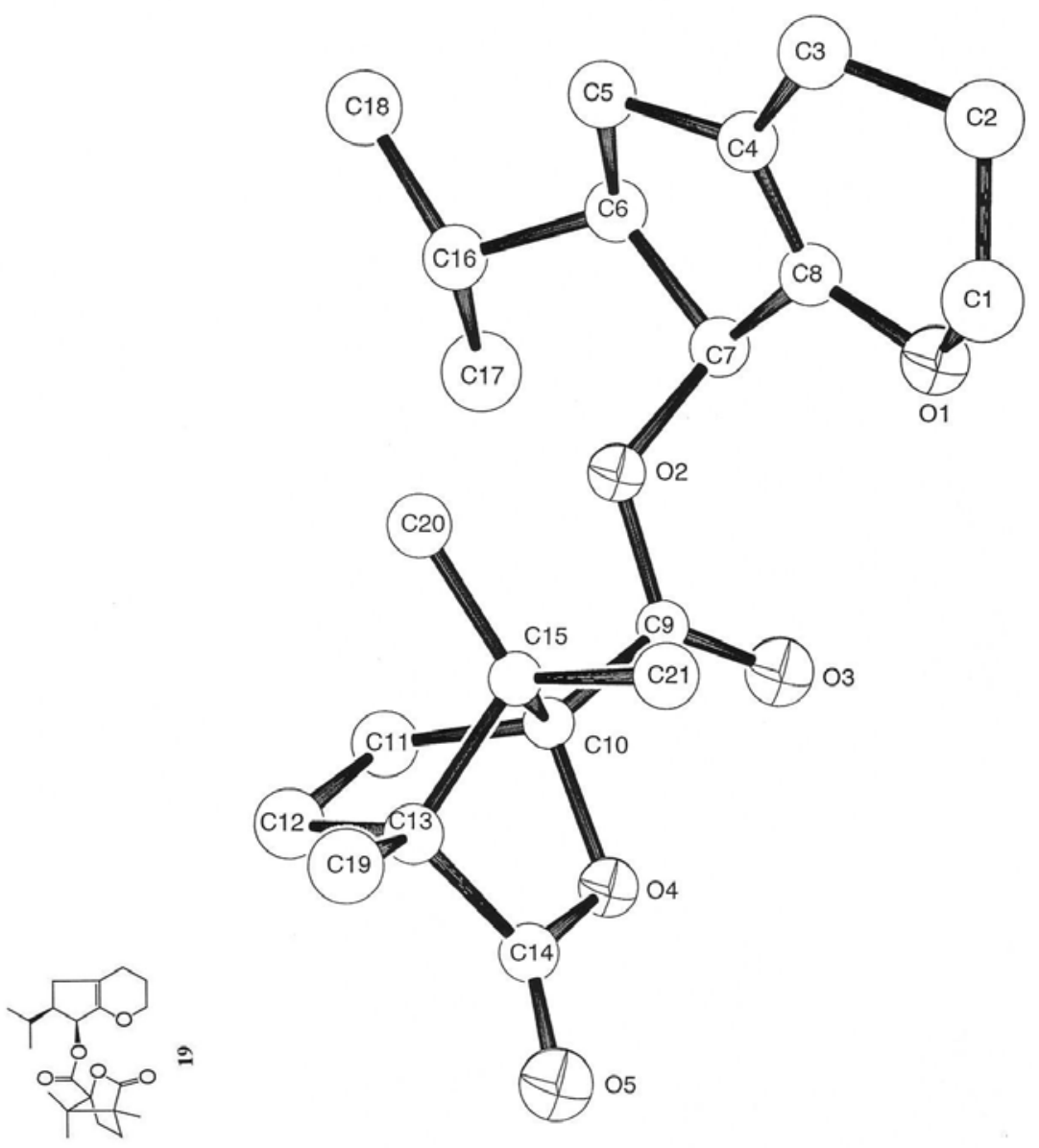